\title{
A search for mid-infrared molecular hydrogen emission from protoplanetary disks ${ }^{\star}$
}

\author{
A. Carmona ${ }^{1,2, \star \star}$, M. E. van den Ancker ${ }^{2}$, Th. Henning ${ }^{1}$, Ya. Pavlyuchenkov ${ }^{1}$, C. P. Dullemond ${ }^{1}$, M. Goto ${ }^{1}$, \\ W. F. Thi ${ }^{3}$, J. Bouwman ${ }^{1}$, and L. B. F. M. Waters ${ }^{4,5}$ \\ 1 Max Planck Institute for Astronomy, Königstuhl 17, 69117 Heidelberg, Germany \\ e-mail: carmona@mpia.de \\ 2 European Southern Observatory, Karl Schwarzschild Strasse 2, 85748 Garching bei München, Germany \\ 3 Royal Observatory Edinburgh, Blackford Hill, Edinburgh EH9 3HJ, UK \\ 4 Astronomical Institute, University of Amsterdam, Kruislaan 403, 1098 SJ Amsterdam, The Netherlands \\ 5 Instituut voor Sterrenkunde, Katholieke Universiteit Leuven, Celestijnenlaan 200B, 3030 Heverlee, Belgium
}

Received 11 May 2007 / Accepted 15 October 2007

ABSTRACT

\begin{abstract}
We report on a sensitive search for mid-infrared molecular hydrogen emission from protoplanetary disks. We observed the Herbig Ae/Be stars UX Ori, HD 34282, HD 100453, HD 101412, HD 104237 and HD 142666, and the T Tauri star HD 319139, and searched for $\mathrm{H}_{2} 0-0 S(2)(J=4-2)$ emission at 12.278 micron and $\mathrm{H}_{2} 0-0 S(1)(J=3-1)$ emission at 17.035 micron with VISIR, ESO-VLT's high-resolution mid-infrared spectrograph. None of the sources present evidence for molecular hydrogen emission at the wavelengths observed. Stringent $3 \sigma$ upper limits to the integrated line fluxes and the mass of optically thin warm gas $(T=$ 150,300 and $1000 \mathrm{~K}$ ) in the disks are derived. The disks contain less than a few tenths of Jupiter mass of optically thin $\mathrm{H}_{2}$ gas at $150 \mathrm{~K}$, and less than a few Earth masses of optically thin $\mathrm{H}_{2}$ gas at $300 \mathrm{~K}$ and higher temperatures. We compare our results to a Chiang \& Goldreich (1997, ApJ, 490, 368, CG97) two-layer disk model of masses $0.02 M_{\odot}$ and $0.11 M_{\odot}$. The upper limits to the disk's optically thin warm gas mass are smaller than the amount of warm gas in the interior layer of the disk, but they are much larger than the amount of molecular gas expected to be in the surface layer. If the two-layer approximation to the structure of the disk is correct, our non-detections are consistent with the low flux levels expected from the small amount of $\mathrm{H}_{2}$ gas in the surface layer. We present a calculation of the expected thermal $\mathrm{H}_{2}$ emission from optically thick disks, assuming a CG97 disk structure, a gas-to-dust ratio of 100 and $T_{\text {gas }}=T_{\text {dust }}$. We show that the expected $\mathrm{H}_{2}$ thermal emission fluxes from typical disks around Herbig Ae/Be stars are of the order of $10^{-16}$ to $10^{-17} \mathrm{erg} \mathrm{s}^{-1} \mathrm{~cm}^{-2}$ for a distance of $140 \mathrm{pc}$. This is much lower than the detection limits of our observations $\left(5 \times 10^{-15} \mathrm{erg} \mathrm{s}^{-1} \mathrm{~cm}^{-2}\right) . \mathrm{H}_{2}$ emission levels are very sensitive to departures from the thermal coupling between the molecular gas and dust in the surface layer. Additional sources of heating of gas in the disk's surface layer could have a major impact on the expected $\mathrm{H}_{2}$ disk emission. Our results suggest that in the observed sources the molecular gas and dust in the surface layer have not significantly departed from thermal coupling $\left(T_{\mathrm{gas}} / T_{\mathrm{dust}}<2\right)$ and that the gas-to-dust ratio in the surface layer is very likely lower than 1000.
\end{abstract}

Key words. stars: emission-line, $\mathrm{Be}$ - stars: pre-main sequence - planetary systems: protoplanetary disks

\section{Introduction}

Circumstellar disks surrounding low- and intermediate-mass stars in their pre-main sequence phase are the locations where planets presumably form. Such protoplanetary disks are composed of gas and dust. Their mass is initially dominated by gas (99\%), specifically by molecular hydrogen $\left(\mathrm{H}_{2}\right)$, which is the most abundant gas species. The dust constitutes only a minor fraction of the total disk mass, however, it is the main source of opacity. Consequently, most of what we know observationally about protoplanetary disks has been inferred from studies of dust emission and scattering (for recent reviews see Henning et al. 2006; Natta et al. 2007; Dullemond et al. 2007; Watson et al. 2007). In order to understand the structure and evolution of protoplanetary disks, it is necessary to study their gaseous content independently from the dust. For example, a basic physical

$\star$ Based on observations collected at the European Southern Observatory, Chile (program ID 076.C-0129(A) and 078.C-0288(A)).

$\star \star$ New address (from October 2007): ISDC, chemin d'Ecogia 16, 1290 Versoix, and Geneva Observatory, University of Geneva, chemin des Maillettes 51, 1290 Sauverny, Switzerland. quantity such as the disk mass is conventionally deduced from dust continuum emission at millimeter wavelengths assuming an interstellar gas-to-dust ratio of 100 (e.g., Beckwith et al. 1990; Henning et al. 1994). If dust is physically processed in the disk, as should be the case in order to form planets, the gas-to-dust ratio must change with time. The disk dissipation time scale another fundamental quantity required to disentangle proposed giant planet formation scenarios (Pollack et al. 1996; Boss et al. 1998) - is deduced from observations of thermal infrared excess emission produced by dust grains (Haisch et al. 2001, 2005). Although recent studies (e.g., Sicilia-Aguilar et al. 2006) suggest a parallel evolution of the dusty and gaseous components, it still remains to be demonstrated that the gaseous disks disappear over the same time scale as the infrared excess.

A variety of spectral diagnostics of the gas disk have been observed from the UV to the millimeter (see reviews by Najita et al. 2007; Dutrey et al. 2007). However, the only diagnostic that is potentially able to probe the warm gas in the regions where giant planets are thought to form is the mid-infrared (mid-IR) emission lines of $\mathrm{H}_{2}$. UV and near-infrared diagnostics only probe the innermost regions of the disk $(R<$ few $\mathrm{AU})$, and $\mathrm{mm}$ 
Table 1. Summary of the stellar properties and previous gas and dust observations.

\begin{tabular}{|c|c|c|c|c|c|c|c|c|c|c|}
\hline Star & Sp.T. & $\log \left(T_{\text {eff }} / \mathrm{K}\right)$ & $\log \left(L / L_{\odot}\right)$ & $\begin{array}{c}V_{\mathrm{rad}} \\
{\left[\mathrm{km} \mathrm{s}^{-1}\right]}\end{array}$ & $\begin{array}{c}d \\
{[\mathrm{pc}]}\end{array}$ & $\begin{array}{c}\mathrm{Age}^{a} \\
{[\mathrm{Myr}]}\end{array}$ & CO sub-mm & $\begin{array}{c}M_{\mathrm{DISK}}{ }^{b} \\
{\left[M_{J}\right]}\end{array}$ & Group I/II ${ }^{c}$ & References \\
\hline UX Ori & A4IVe & 3.92 & 1.68 & 18 & 340 & 5 & $\overline{\text { D05 }}$ & 16 & II & A04, D05, D99 \\
\hline HD 34282 & A3Vne & 3.94 & 1.27 & 16 & 400 & 7 & D05 & 100 & I & $\mathrm{A} 04, \mathrm{P} 03$ \\
\hline HD 100453 & A9Ve & 3.87 & 0.90 & 17 & 112 & $>10$ & $\ldots$ & 21 & I & A04, A05, HIP \\
\hline HD 101412 & B9.5Ve & 4.02 & 1.40 & 17 & 160 & $>8$ & $\ldots$ & $\ldots$ & II & A04, VP, D99 \\
\hline HD 104237 & $\mathrm{~A} 4 \mathrm{IVe}+\mathrm{sh}$ & 3.92 & 1.54 & 13 & 116 & 2 & $\ldots$ & 7.8 & II & $\mathrm{A} 04, \mathrm{~A} 05, \mathrm{~V} 98$ \\
\hline HD 142666 & $\mathrm{~A} 8 \mathrm{Ve}$ & 3.88 & 1.13 & 3 & 145 & 6 & D05 & 16 & II & A04, DU97, D99 \\
\hline HD $319139^{d}$ & $\mathrm{~K} 5 \mathrm{Ve}+\mathrm{K} 7 \mathrm{Ve}$ & $3.64+3.61$ & 0.44 & -7 & 145 & $2^{e}$ & $\ldots$ & $47^{f}$ & CTTS & S04, D99 \\
\hline
\end{tabular}

${ }^{a}$ Ages derived from the H-R diagram employing the pre-main-sequence evolutionary tracks of Palla \& Stahler (1993). ${ }^{b}$ Total disk gas masses are derived from dust continuum emission at millimeter wavelengths assuming a gas-to-dust ratio of $100 .{ }^{c}$ Group of each Herbig Ae/Be star disk according to the classification scheme of Meeus et al. (2001). Group I sources - flared disks - are sources with a rising mid-IR spectral energy distribution (SED) and Group II sources - self-shadowed disks - are sources with a flat SED (Dullemond et al. 2002). CTTS: Classical T Tauri star. ${ }^{d}$ Spectroscopic binary of period 2.4 days [S04]. ${ }^{e}$ We assume that both components have the same luminosity. ${ }^{f}$ Disk mass by Jensen et al. (1996) scaled to our adopted distance of $145 \mathrm{pc}$.

References: [A04] Acke et al. (2004); [A05] Acke et al. (2005); [D05] Dent et al. (2005); [D99] de Zeeuw et al. (1999); [DU97] Dunkin et al. (1997); [HIP] Hipparcos catalogue; [P03] Piétu et al. (2003); [V98] van den Ancker et al. (1998); [VP] van der Plas et al. (in prep.); [S04] Stempels \& Gahm (2004).

and sub-mm diagnostics are limited to probe the cold outermost regions of the disk $(R>10 \mathrm{AU})$.

Molecular hydrogen is by far the most abundant molecular species in protoplanetary disks. Unfortunately, $\mathrm{H}_{2}$ is one of the most challenging molecules to detect. Since $\mathrm{H}_{2}$ is a homonuclear molecule, it lacks a permanent dipole moment and its transitions are thus electric quadrupole in nature. The small Einstein coefficients, characteristic of the quadrupole transitions, imply that $\mathrm{H}_{2}$ emission features are very weak. In addition, in the case of protoplanetary disks, the $\mathrm{H}_{2}$ lines are not sensitive to the warm gas in the optically thick regions where the dust and gas are at equal temperature. Practical observational challenges also have to be faced. The mid-IR $\mathrm{H}_{2}$ emission from the disk needs to be detected on the top of a strong mid-IR continuum. From the ground, the mid-infrared windows are strongly affected by sky and instrument background emission, and the $\mathrm{H}_{2}$ transitions at 12 and $17 \mu \mathrm{m}$ lie close to atmospheric absorption lines highly dependent on atmospheric conditions. The advent of high spectral resolution spectrographs mounted on larger aperture telescopes, allows for the first time the study of $\mathrm{H}_{2}$ emission from the ground, but the search is still limited to bright targets. From space, the problems of atmosphere absorption are alleviated and the $J=2-0$ feature at $28 \mu \mathrm{m}$ is visible. However, the beam sizes are large and the spectral resolution of space mid-infrared spectrographs are usually low when compared to ground-based facilities, therefore, they are not very appropriate for small lineto-continuum ratios.

$\mathrm{H}_{2}$ emission from protoplanetary disks in the mid-IR has been reported from ISO observations (Thi et al. 2001). However, subsequent ground-based efforts (Richter et al. 2002; Sheret et al. 2003; Sako et al. 2005) did not confirm the ISO detections. $\mathrm{H}_{2}$ emission in the mid-IR has been searched towards debris disks using Spitzer (Hollenbach et al. 2005; Pascucci et al. 2006; Chen et al. 2006) with no detection reported. Most recently, Bitner et al. (2007) and Martin-Zaïdi et al. (2007) reported the detection of mid-IR $\mathrm{H}_{2}$ emission in two Herbig Ae/Be stars (AB Aur and HD 97048) from the ground, and Lahuis et al. (2007) reported the detection of mid-IR $\mathrm{H}_{2}$ emission in 6 T Tauri stars with Spitzer.

Here, we report on a sensitive search for molecular hydrogen emission from protoplanetary disks. We observed six southern nearby $(d<400 \mathrm{pc})$ Herbig Ae/Be stars and one $\mathrm{T}$ Tauri star, employing the Very Large Telescope (VLT) imager and spectrometer for the mid-infrared (VISIR; Lagage et al. 2004) ${ }^{1}$, ESO's VLT mid-infrared high-resolution spectrograph, and searched for $\mathrm{H}_{2} 0-0 S(1)(J=3-1)$ emission at $17.035 \mu \mathrm{m}$ and $\mathrm{H}_{2} 0-0 S(2)(J=4-2)$ emission at $12.278 \mu \mathrm{m}$. The paper is organized as follows: in Sect. 2 we present the sample studied, and the details of how the observations were performed. In Sect. 3 we discuss the data reduction. In Sect. 4 we deduce upper limits to the $\mathrm{H}_{2}$ fluxes, and using the optically thin approximation, we derive upper limits to the mass of warm $(150-1000 \mathrm{~K})$ gas in the disks. In Sect. 5 we discuss our results in the context of the Chiang \& Goldreich (1997) two-layer disk model. Finally, we summarize our results and conclusions in Sect. 6.

\section{Observations}

Target selection. We selected a sample of well known nearby Herbig Ae/Be and T Tauri stars based on evidence of large disk reservoirs. The targets have either reported detections of cold $\mathrm{CO}$ gas at (sub)-mm wavelengths, or dust continuum emission at mm wavelengths. We chose stars with $12 \mu \mathrm{m}$ continuum fluxes $>0.5 \mathrm{Jy}$ (otherwise too faint for acquisition with VISIR) and $<25 \mathrm{Jy}$ (hard to detect weak lines on top of a strong continuum). The physical properties of the targets are compiled in Table 1.

Observations. The sources were observed in the first semester of 2006 and 2007 with VISIR, a combined imager and spectrograph designed for observations in the $N(\approx 8-13 \mu \mathrm{m})$ and $Q$ bands $(\approx 16.5-24.5 \mu \mathrm{m})$ (Lagage et al. 2004), mounted at the ESO-VLT Melipal telescope in Cerro Paranal, Chile. The $\mathrm{H}_{2}$ 0-0 $S(1)(J=3-1)$ line at $17.035 \mu \mathrm{m}$ was observed in the high-resolution long-slit mode with a 0.4 arcsec slit, giving a spectral resolution $R \approx 21000$, or $14 \mathrm{~km} \mathrm{~s}^{-1}$. The $\mathrm{H}_{2} 0-0 S(2)(J=4-2)$ line at $12.278 \mu \mathrm{m}$ was observed in the high-resolution echelle mode with a 0.4 arcsec slit, giving a spectral resolution $R \approx 20000$, or $15 \mathrm{~km} \mathrm{~s}^{-1}$.

The total integration time in each line was $1 \mathrm{~h}$. The slit was oriented in the North-South direction. Sky background was subtracted by chopping the telescope by $\sim 8^{\prime \prime}$ in the direction of the slit. Asymmetrical thermal background of the telescope was subtracted by nodding the telescope by $\sim 8^{\prime \prime}$ in the direction of the

\footnotetext{
1 http://www . eso.org/instruments/visir
} 
Table 2. Summary of the observations.

\begin{tabular}{|c|c|c|c|c|c|c|c|c|c|c|c|}
\hline \multirow{2}{*}{$\begin{array}{l}\text { Star } \\
\text { UX Ori }\end{array}$} & \multirow{2}{*}{$\left.\begin{array}{c}\lambda \\
{[\mu \mathrm{m}]}\end{array}\right]$} & \multirow{2}{*}{$\begin{array}{l}\text { Date } \\
11 \text { January } 2006\end{array}$} & \multirow{2}{*}{$\begin{array}{c}\begin{array}{c}\text { U.T. } \\
\text { [hh:mm] }\end{array} \\
02: 28\end{array}$} & \multirow{2}{*}{$\begin{array}{c}t_{\exp } \\
{[\mathrm{s}]} \\
3600\end{array}$} & \multirow{2}{*}{$\begin{array}{c}\text { Airmass }^{a} \\
1.0-1.2\end{array}$} & \multirow{2}{*}{$\begin{array}{c}\begin{array}{c}V_{\oplus \mathrm{rad}^{b}}{ }^{-1} \\
{\left[\mathrm{~km} \mathrm{~s}^{-1}\right]}\end{array} \\
2.06\end{array}$} & \multicolumn{2}{|c|}{ Calibrator $^{c}$} & \multirow{2}{*}{$\begin{array}{r}t_{\text {exp }} \\
{[\mathrm{s}]} \\
1000\end{array}$} & \multicolumn{2}{|c|}{ Airmass } \\
\hline & & & & & & & HD 36167 & $(\mathrm{P})(\mathrm{F})$ & & $1.1(\mathrm{P})$ & $1.2(\mathrm{~F})$ \\
\hline & 17.035 & 4 January 2007 & 01:54 & 3600 & $1.0-1.0$ & 4.50 & HD 25025 & (P) & 600 & $1.0(\mathrm{P})$ & \\
\hline HD 34282 & 12.278 & 10 January 2006 & 04:01 & 3600 & $1.0-1.5$ & 2.17 & HD 36167 & $(\mathrm{P})(\mathrm{F})$ & 1000 & $1.1(\mathrm{P})$ & $1.7(\mathrm{~F})$ \\
\hline \multirow{2}{*}{ HD 100453} & 12.278 & 22 March 2006 & $07: 27$ & 3600 & $1.2-1.5$ & 24.45 & HD 89388 & $(\mathrm{P})(\mathrm{F})$ & 600 & $1.5(\mathrm{P})$ & $2.0(\mathrm{~F})$ \\
\hline & 17.035 & 27 March 2006 & $06: 26$ & 3600 & $1.3-1.7$ & 22.96 & HD 89388 & (P) & 1000 & $1.5(\mathrm{P})$ & \\
\hline \multirow[t]{2}{*}{ HD 101412} & 12.278 & 30 March 2006 & 04:40 & 3600 & $1.2-1.4$ & 23.82 & HD 91056 & $(\mathrm{P})(\mathrm{F})$ & 600 & $1.3(\mathrm{P})$ & $1.7(\mathrm{~F})$ \\
\hline & 17.035 & 30 March 2006 & 01:27 & 3600 & $1.3-1.2$ & 24.02 & HD 91056 & (F) & 1000 & & $1.3(\mathrm{~F})$ \\
\hline \multirow[t]{2}{*}{ HD 104237} & 12.278 & 10 February 2006 & $05: 43$ & 3600 & $1.7-1.7$ & 26.09 & HD 92305 & $(\mathrm{P})(\mathrm{F})$ & 600 & $1.7(\mathrm{P})$ & $1.8(\mathrm{~F})$ \\
\hline & 17.035 & 12 February 2006 & $06: 36$ & 3600 & $1.6-1.7$ & 26.15 & HD 92305 & (F) & 1000 & & $1.8(\mathrm{~F})$ \\
\hline HD 142666 & 17.035 & 28 February 2006 & $06: 28$ & 3600 & $1.1-1.0$ & 27.06 & HD 169916 & (P) & 1000 & $1.1(\mathrm{P})$ & $\ldots$ \\
\hline HD 319139 & 17.035 & 30 March 2006 & $08: 30$ & 3600 & $1.1-1.0$ & 22.47 & HD 169916 & (P) & 1000 & $1.2(\mathrm{P})$ & $\ldots$ \\
\hline
\end{tabular}

${ }^{a}$ The airmass interval is given from the beginning to the end of the observations. ${ }^{b} V_{\oplus \text { rad }}$ is the expected velocity shift of the spectra due to the reflex motion of the Earth around the Sun and the radial velocity of the star. ${ }^{c}$ The standard stars were observed prior $(\mathrm{P})$ and/or immediately following $(\mathrm{F})$ the science observations.

slit. For correcting the spectra for telluric absorption and obtaining the absolute flux calibration, spectroscopic standard stars at close airmasses to that of the science targets were observed immediately preceding and following the $12 \mu \mathrm{m}$ exposure, and preceding or following the $17 \mu \mathrm{m}$ exposure ${ }^{2}$. Finally, we tried as much as possible to observe the stars at a moment of high velocity shift between the telluric absorption feature at 12.27 microns and the $\mathrm{H}_{2} \mathrm{~S}(2)$ line. This effect is due to the reflex motion of the Earth around the Sun. The typical PSF FWHM measured in the continuum was $0.5^{\prime \prime}$ ( $\sim 4$ pixels using the VISIR pixel scale of $0.127^{\prime \prime} / \mathrm{pixel}$ ) equivalent to a spatial resolution of $50 \mathrm{AU}$ for a source located at $100 \mathrm{pc}$. A summary of the observations is presented in Table 2.

\section{Data reduction}

VISIR raw data consists of a collection of data cubes, in which each data cube is associated with data taken in one nod position $^{3}$. The odd planes in the data cube correspond to a halfchop cycle frame, the even planes correspond to the average of the difference of all preceding half-chop cycles (i.e. $A_{i}=$ $\left.A_{1}-B_{1}+A_{2}-B_{2}+\ldots+A_{i}-B_{i} / i\right)$. The last plane corresponds to the average of all half-cycle difference images.

The first step in the data reduction process was to recover the individual half chop cycle frames from the data cube, and to guarantee that all of them have the same wavelength in the same row of pixels (in each 2D spectrum, the spatial direction is along the columns and the dispersion direction is along the rows). In each 2D half-chop cycle frame, at the position of the target spectrum, a cut with a size of 20 pixels in the spatial direction was made. From this cut, a spectrum of the atmospheric emission was obtained by the sum of the counts in the spatial direction (see uppermost right panel in Figs. 1 and 2). This atmospheric emission spectrum was rebinned by a factor of 50 (i.e. 50 pixels where previously we had 1). Using as reference the atmospheric

${ }^{2}$ The $12 \mu \mathrm{m}$ window is much more affected by telluric absorption than the $17 \mu \mathrm{m}$ window. In order to be able to select the standard star observation that provides the best telluric correction, observations of the $\mathrm{H}_{2} \mathrm{~S}(2)$ line require the observation of a telluric standard preceding and following the science observation. The 17 micron window is less affected than the 12 micron window by telluric absorption, therefore, only one observation of a telluric standard (following or preceding the science observation) is required.

3 The sequence of the observations is ABBA, where A and B correspond to two different nod positions. emission spectrum from the first half-chop cycle of the first nod, the offset that each half-chop cycle raw frame required for having the center of the sky emission lines at the same pixel position as the reference frame was determined. The offset was given by the difference between the center of the Gaussian fitted to one sky emission line in the reference spectra, and the center of the Gaussian fitted to the same sky line in the atmospheric emission spectrum of the half-chop cycle frame to be corrected. The typical offsets were a fraction of a pixel (before rebinning), and did not depend on the reference sky line. Then, each 2D raw halfchop cycle frame was rebinned by a factor of 50 (i.e. 50 pixels where previously we had 1) in the dispersion direction, and it was shifted - in the dispersion direction - by the offset found. Finally, the obtained 2D frame was rebinned to the original size of $256 \times 256$ pixels. This procedure corrected small differences in $2 \mathrm{D}$ half-chop cycles introduced by variations in the grating position within the instrument.

Thereafter, with the corrected half-chop frames, the VISIR data cubes were re-built and processed with the VISIR pipeline (Lundin 2006). Bad pixels were detected in the half-cycle frames when their number of counts exceeded the limit of 65000 . They were cleaned by interpolation with neighboring pixels. In the case of the $17 \mu \mathrm{m}$ data (i.e. taken in the long-slit mode), the 2D frames were corrected for distortion by interpolating the analytical optical distortion (see description in the VISIR user's manual; Siebenmorgen et al. 2006) and the value from the source pixels. The $2 \mathrm{D}$ frames in each nod position were created by averaging all the half-cycle difference images in the data cube. The nodding images were produced by averaging the image in the two nod positions $A$ and $(-B)$, and dividing the result by $2 \times$ DIT, where DIT is the detector integration time ${ }^{4}$. The nodded images are jittered and were thus shifted and added to form the final combined image using the offsets stored in the file header.

The wavelength calibration was performed with the following procedure. The first half-cycle from the first nod position was "collapsed", producing a 1-dimensional spectrum of the atmosphere. The centers of the atmospheric emission lines (in pixels) were found by fitting Gaussians to their profiles in a HITRAN model spectrum of Paranal's atmosphere emission (for details of the model, see Lundin 2006). The dispersion relation was found by a second degree polynomial fit between the centers of the atmospheric lines in the observed and model spectrum.

\footnotetext{
4 The factor 2 is due to the fact that the on-source and the off-source images both contribute to the whole DIT.
} 


$$
\mathrm{H}_{2} \mathrm{O}-\mathrm{O} \mathrm{S}(2) \mathrm{J}=4-2 \lambda=12.278 \mu \mathrm{m}
$$
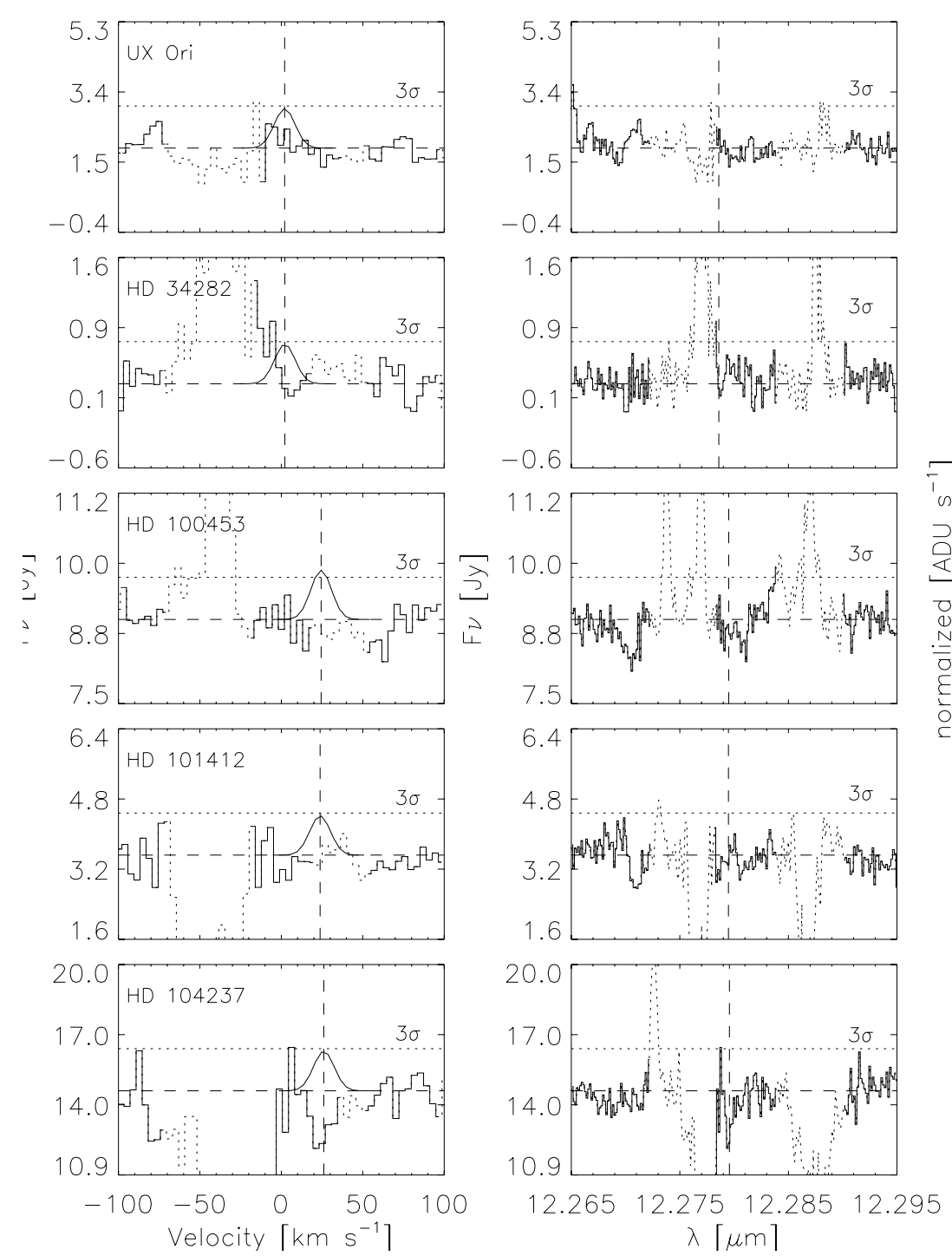
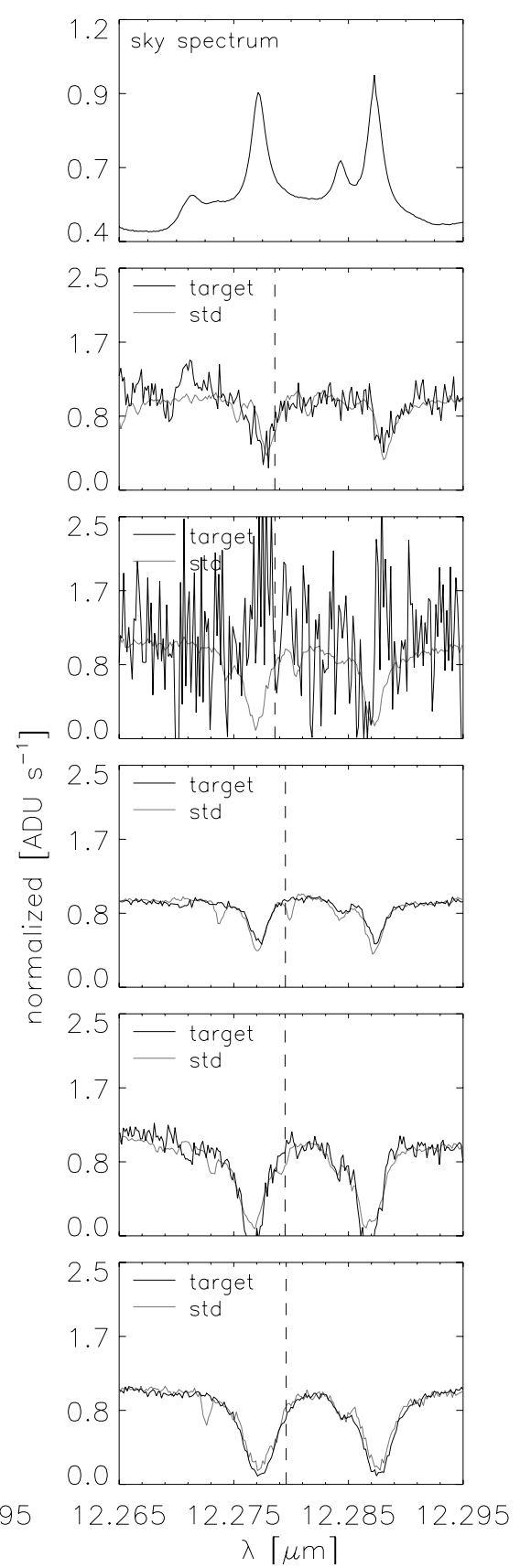

Fig. 1. Spectra obtained for the $\mathrm{H}_{2} 0-0 \mathrm{~S}(2)(J=4-2)$ line at $12.278 \mu \mathrm{m}$. The left panel shows a zoom to the -100 to $100 \mathrm{~km} \mathrm{~s}^{-1}$ interval of the atmospheric corrected spectra. A Gaussian of $F W H M=15 \mathrm{~km} \mathrm{~s}^{-1}$ and integrated line flux equal to the line-flux upper limits obtained is overplotted at the expected velocity shifted location (vertical dashed line, see Table 2). The central panel shows the full corrected spectra. Dotted lines show spectral regions strongly affected by telluric or standard star absorption features. The right panel shows the continuum normalized spectra of the standard star and the target before telluric correction. The uppermost right panel displays the sky spectrum from a half-chop cycle. The spectra are not corrected for the radial velocity of the targets.

The spectrum at each wavelength was extracted from the 2D combined image by summing the pixels in the spatial direction inside the PSF. In the case of the long-slit $17 \mu \mathrm{m}$ data, one spectrum was extracted for each of the nod positions present in the final 2D combined image. The final extracted spectrum is the sum of these three spectra. The telluric correction was performed by dividing the extracted spectra by the extracted spectra of the standard star. Small shifts (of a fraction of a pixel) on the spectra of the standard star in the wavelength direction were applied until the best signal to noise spectra (i.e. best telluric correction) were found. The spectral flux calibration was made by multiplying the telluric-corrected science spectrum with a model spectrum (Cohen et al. 1999) of the standard star ${ }^{5}$.

In the case of the observations of the $\mathrm{H}_{2} \mathrm{~S}(2)$ line in HD 34282, HD 100453 and HD 101412 the telluric standard stars showed photospheric absorption lines close to the location of the $\mathrm{H}_{2}$ line. In the case of the HD 34282 observation, the photospheric absorption is located outside the region where the $\mathrm{H}_{2}$ line is expected (after correction by the velocity shift).

\footnotetext{
5 The catalogue of spectroscopic standard stars in the mid-IR is available at www. eso.org/VISIR/catalogue
} 


$$
H_{2} O-O S(1)(J=3-1) \lambda=17.035 \mu m
$$
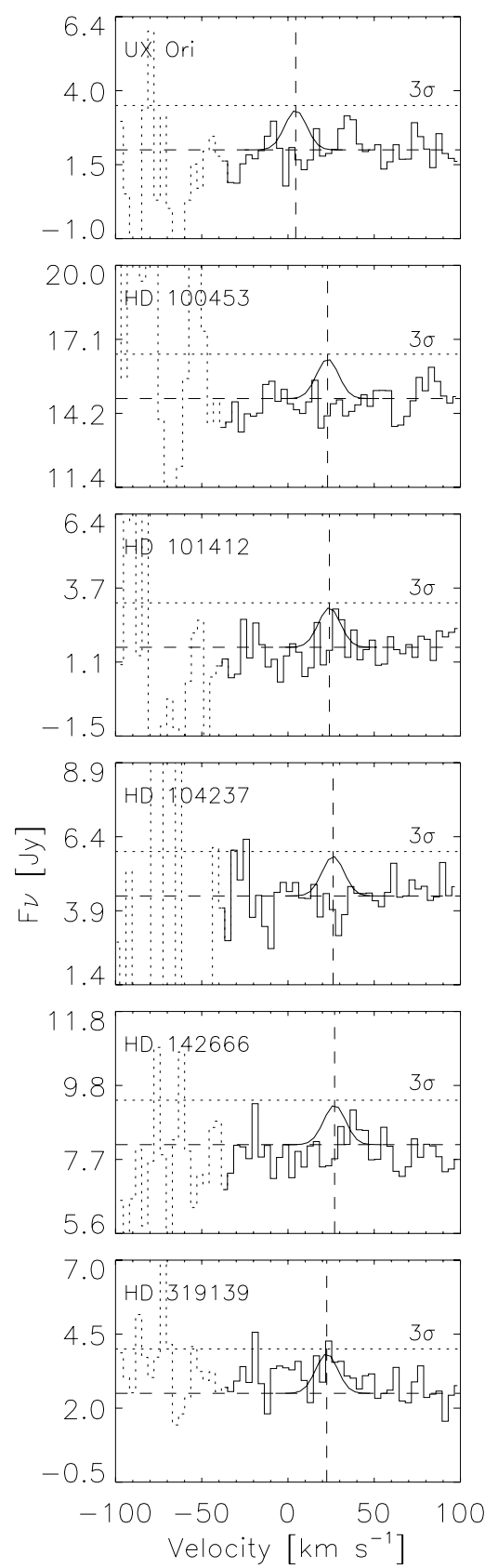
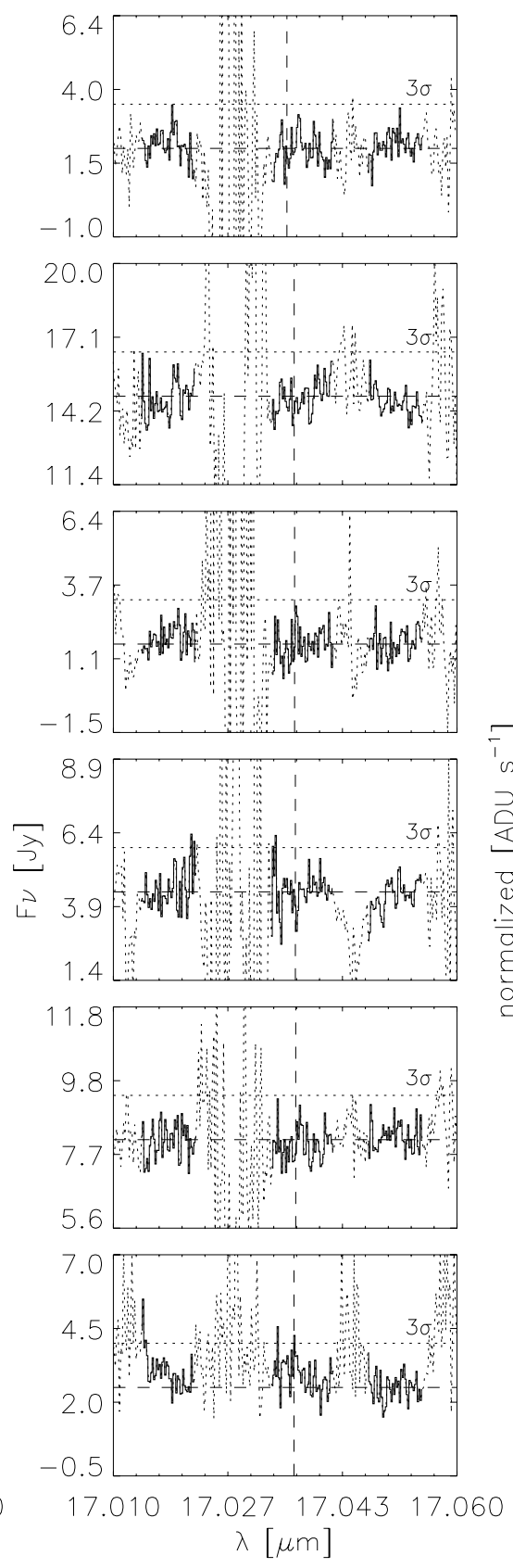
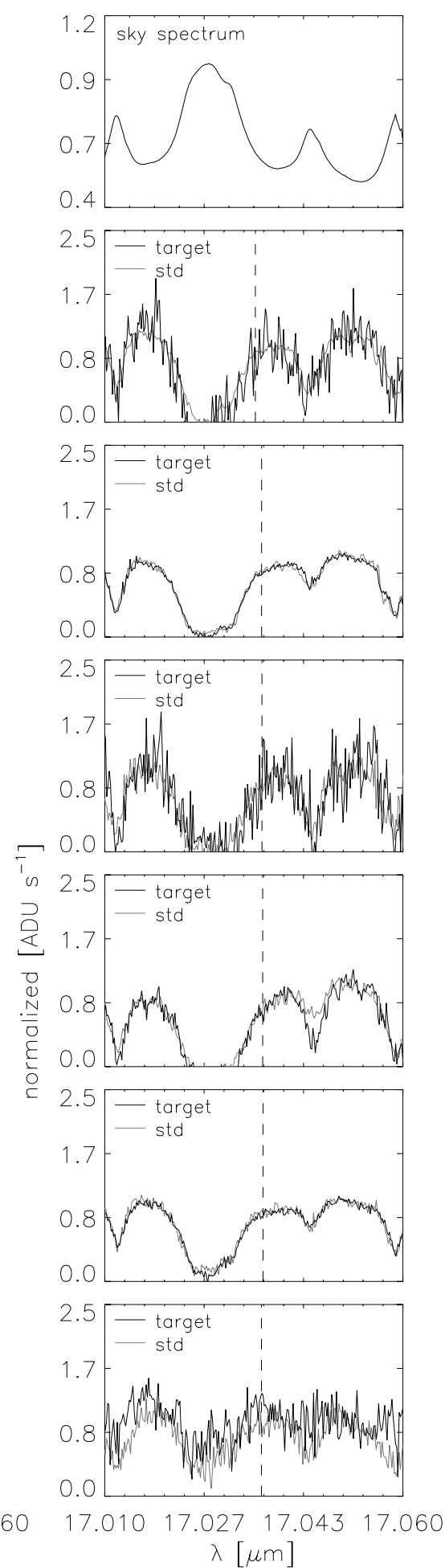

Fig. 2. Spectra obtained for the $\mathrm{H}_{2} 0-0 \mathrm{~S}(1)(J=3-1)$ line at $17.035 \mu \mathrm{m}$. The left panel shows a zoom to the -100 to $100 \mathrm{~km} \mathrm{~s}^{-1}$ interval of the atmospheric corrected spectra. A Gaussian of $F W H M=15 \mathrm{~km} \mathrm{~s}^{-1}$ and integrated line flux equal to the line-flux upper limits obtained is overplotted at the expected velocity shifted location (vertical dashed line, see Table 2). The central panel shows the full corrected spectra. Dotted lines show spectral regions strongly affected by telluric or standard star absorption features. The right panel shows the continuum normalized spectra of the standard star and the target before telluric correction. The uppermost right panel displays the sky spectrum from a half-chop cycle. The spectra are not corrected for the radial velocity of the targets.

However, in the cases of HD 100453 and HD 101412 the expected location of the $\mathrm{S}(2) \mathrm{H}_{2}$ line partially overlaps with the location of the photospheric absorption feature in the spectrum of the standard star (see Fig. 3). This absorption feature is observed superimposed on the broad telluric absorption line at 12.277 micron. To avoid the error induced by this feature in the telluric corrected spectra, we removed this absorption feature from the standard star spectra by a Gaussian fit to the 
Table 3. Upper flux limits.

\begin{tabular}{|c|c|c|c|c|c|c|}
\hline \multirow[b]{3}{*}{ Star } & \multicolumn{3}{|c|}{ 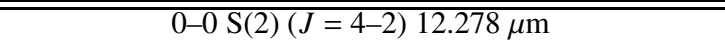 } & \multicolumn{3}{|c|}{ 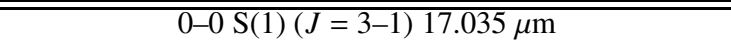 } \\
\hline & \multirow{2}{*}{$\begin{array}{c}\text { Continuum }^{a} \\
F_{v} \\
{[\mathrm{Jy}]}\end{array}$} & \multicolumn{2}{|c|}{$\mathrm{LW}=15 \mathrm{~km} \mathrm{~s}^{-1}$} & \multirow{2}{*}{$\begin{array}{c}\text { Continuum }^{a} \\
F_{v} \\
{[\mathrm{Jy}]}\end{array}$} & \multicolumn{2}{|c|}{$\mathrm{LW}=15 \mathrm{~km} \mathrm{~s}^{-1}$} \\
\hline & & $\begin{array}{c}\text { Line flux } \\
{\left[\times 10^{-14} \mathrm{erg} \mathrm{s}^{-1} \mathrm{~cm}^{-2}\right]}\end{array}$ & $\begin{array}{l}\text { Line luminosity } \\
\quad\left[\log L / L_{\odot}\right]\end{array}$ & & $\begin{array}{c}\text { Line flux } \\
{\left[\times 10^{-14} \mathrm{erg} \mathrm{s}^{-1} \mathrm{~cm}^{-2}\right]}\end{array}$ & $\begin{array}{l}\text { Line luminosity } \\
\quad\left[\log L / L_{\odot}\right]\end{array}$ \\
\hline$\overline{\text { UX Ori }}$ & $1.9(1.1)$ & $<1.4$ & $<-4.3$ & $2.0(1.5)$ & $<1.3$ & $<-4.3$ \\
\hline HD 34282 & $0.3(0.4)$ & $<0.5$ & $<-4.6$ & $\ldots$ & $\ldots$ & $\ldots$ \\
\hline HD 100453 & $9.0(0.7)$ & $<0.9$ & $<-5.5$ & $14.8(1.7)$ & $<1.5$ & $<-5.2$ \\
\hline HD $101412^{b}$ & $3.5(1.0)$ & $<1.2$ & $<-5.0$ & $1.6(1.6)$ & $<1.4$ & $<-5.0$ \\
\hline HD 104237 & $14.6(1.8)$ & $<2.2$ & $<-5.0$ & $4.4(1.5)$ & $<1.3$ & $<-5.3$ \\
\hline HD 142666 & $\ldots$ & $\ldots$ & $\ldots$ & $8.0(1.2)$ & $<1.1$ & $<-5.1$ \\
\hline HD 319139 & $\ldots$ & $\ldots$ & $\ldots$ & $2.5(1.5)$ & $<1.3$ & $<-5.1$ \\
\hline
\end{tabular}

${ }^{a}$ In brackets the $3 \sigma$ error in the continuum flux. ${ }^{b}$ Source also observed with Spitzer by Lahuis et al. (2007). They report a non-detection of the $\mathrm{H}_{2} \mathrm{~S}(1)$ and $\mathrm{H}_{2} \mathrm{~S}(2)$ lines with $1 \sigma$ upper limits of $0.9 \times 10^{-14} \mathrm{erg} \mathrm{s}^{-1} \mathrm{~cm}^{-2}$ assuming a line width of $500 \mathrm{~km} \mathrm{~s}^{-1}$.
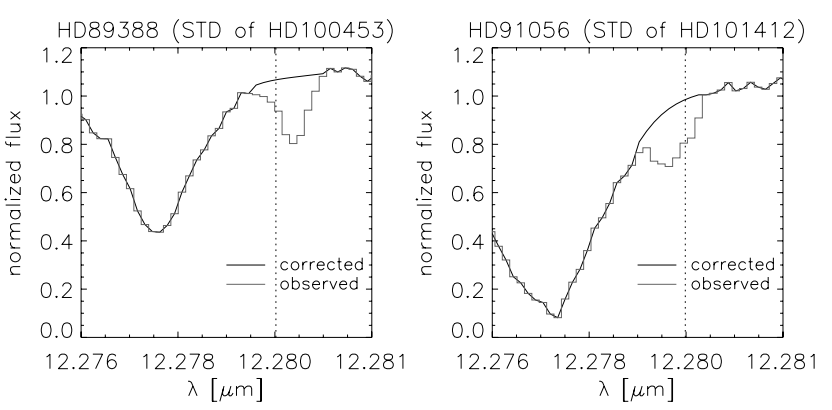

Fig. 3. Correction for photospheric absorption features in the spectrum of the telluric standard (STD) of HD 100453 and HD 101412. In grey the observed STD spectrum, in black the corrected STD spectrum. The photospheric feature was removed by a Gaussian fit to the telluric line at $12.277 \mu \mathrm{m}$. The vertical dotted line displays the expected location of the $\mathrm{S}(2) \mathrm{H}_{2}$ line in the science spectrum.

underlying broad atmospheric absorption feature at 12.277 micron (see Fig. 3). After this correction, the science spectra were divided by the telluric standard and flux calibrated in the conventional way.

\section{Results}

In Figs. 1 and 2 we present a compilation of the obtained spectra. In the left panel, the atmosphere-corrected flux calibrated spectra in wavelength scale measured by the velocities in $\mathrm{km} \mathrm{s}^{-1}$ are shown. In the central panel, the full corrected spectra are presented. In the right panel, for reference, the normalized spectra of the science target and the standard star are displayed. None of the observed sources show evidence for $\mathrm{H}_{2}$ emission at 12 or $17 \mu \mathrm{m}$.

Line flux upper limits. In the flux-calibrated science spectra, the standard deviation $(\sigma)$ of the continuum flux was calculated in regions less influenced by telluric absorption, photospheric features of the standard star and close to the features of interest. In Figs. 1 and 2, the horizontal dotted line shows the $3 \sigma$ limits of the derived continuum flux. $3 \sigma$ upper limits to the integrated line flux were calculated by multiplying the $3 \sigma$ flux with the instrument resolution line width ${ }^{6}$ of $15 \mathrm{~km} \mathrm{~s}^{-1}$.

${ }^{6}$ Here we assume a pole-on disk and that the true-line width is narrower than the instrumental line width of $15 \mathrm{~km} \mathrm{~s}^{-1}$ (i.e. the spectral line is not resolved). If the line is broader, the sensitivity limits in Table 3 decrease proportionally with the $F W H M$ (in $\mathrm{km} \mathrm{s}^{-1}$ ) following $F=F_{15 \mathrm{~km} \mathrm{~s}^{-1}} *(F W H M / 15)$.
In Table 3, a summary of the upper flux limits is presented. In Figs. 1 and 2 a Gaussian line of $F W H M=15 \mathrm{~km} \mathrm{~s}^{-1}$ and an integrated flux equal to the upper flux limit is overplotted on each observed spectrum. The typical sensitivity limit of our observations is a line flux of $10^{-14} \mathrm{erg} \mathrm{s}^{-1} \mathrm{~cm}^{-2}$. Our flux limits are of the order of magnitude of the $\mathrm{H}_{2} \mathrm{~S}(1)$ and $\mathrm{S}(2)$ lines fluxes (1.1 and $0.53 \times 10^{-14} \mathrm{erg} \mathrm{s}^{-1} \mathrm{~cm}^{-2}$, respectively) reported by Bitner et al. (2007) for $\mathrm{AB}$ Aur and the $\mathrm{H}_{2}$ line fluxes of $0.33-1.70 \times$ $10^{-14} \mathrm{erg} \mathrm{s}^{-1} \mathrm{~cm}^{-2}$ reported for the $\mathrm{H}_{2} \mathrm{~S}(2)$ line in the observations by Lahuis et al. (2007). Here, we should note that the observations of AB Aur were performed with TEXES, which provides a spectral resolution of 100000 , increasing the line-tocontinuum contrast compared with our observations.

Upper limits on the optically thin warm gas disk mass. Under the assumption that the $\mathrm{H}_{2}$ emission and the emission of the accompanying dust are optically thin, that the emitting $\mathrm{H}_{2}$ is in local thermodynamical equilibrium, and that the source size is equal or smaller than VISIR's beam size, we derived upper limits to the $\mathrm{H}_{2}$ mass as a function of the temperature employing (Thi et al. 2001)

$M_{\mathrm{gas}}=f \times 1.76 \times 10^{-20} \frac{4 \pi d^{2} F_{\mathrm{ul}}}{E_{\mathrm{ul}} A_{\mathrm{ul}} x_{u}(T)} M_{\odot}$,

where $F_{\mathrm{ul}}$ is the upper limit to the integrated line flux, $d$ is the distance in pc to the star, $E_{\mathrm{ul}}$ is the energy of the transition, $A_{\mathrm{ul}}$ is the Einstein coefficient of the $J=u-l$ transition $^{7}$ and $x_{u}$ is the population of the level $u$ at the excitation temperature $T$ in LTE; $f$ is the conversion factor required for deriving the total gas mass from the $\mathrm{H}_{2}$-ortho or $\mathrm{H}_{2}$-para mass determined. Since $M_{\mathrm{H}_{2}}=M_{\mathrm{H}_{2} \text { ortho }}+M_{\mathrm{H}_{2} \text { para }}, f=1+$ ortho/para for the $\mathrm{S}$ (2) line (a $\mathrm{H}_{2}$-para transition) and $f=1+1 /$ (ortho/para) for the $\mathrm{S}(1)$ line (a $\mathrm{H}_{2}$-ortho transition). The equilibrium ortho-para ratio at the temperature $T$ was computed using Eq. (1) of Takahashi (2001). In Table 4, we present our results.

The $\mathrm{H}_{2} \mathrm{~S}(1)$ and $\mathrm{S}(2)$ line fluxes constrain the amount of optically thin hot and warm $\mathrm{H}_{2}$ gas. The disks contain less than a few tenths of Jupiter mass of optically thin $\mathrm{H}_{2}$ at $150 \mathrm{~K}$, and less than a few Earth masses of optically thin $\mathrm{H}_{2}$ at $300 \mathrm{~K}$ and higher temperatures. In Fig. 4 we present the mass of optically thin $\mathrm{H}_{2}$ as a function of the temperature derived from a $\mathrm{S}(1)$ and a $\mathrm{S}(2)$ line with a flux $10^{-14} \mathrm{erg} \mathrm{s}^{-1} \mathrm{~cm}^{-2}$ for a source at a distance of $140 \mathrm{pc}$. This mass is the minimum amount of optically thin $\mathrm{H}_{2}$ that would produce a detectable $\mathrm{H}_{2}$ line in our observations

\footnotetext{
$74.76 \times 10^{-10} \mathrm{~s}^{-1}$ for the $\mathrm{S}(1)$ line $J=3-1$ transition, $2.76 \times 10^{-9} \mathrm{~s}^{-1}$ for the $S(2)$ line $J=4-2$ transition (Wolniewicz et al. 1998).
} 
Table 4. Upper warm $\mathrm{H}_{2}$ mass limits.

\begin{tabular}{|c|c|c|c|c|c|c|}
\hline \multirow[b]{3}{*}{$\underline{\text { Star }}$} & \multicolumn{6}{|c|}{ Upper mass limits in $M_{J} \sim 10^{-3} M_{\odot}$} \\
\hline & \multicolumn{3}{|c|}{$12 \mu \mathrm{m}$} & \multicolumn{3}{|c|}{$17 \mu \mathrm{m}$} \\
\hline & $150 \mathrm{~K}$ & $300 \mathrm{~K}$ & $1000 \mathrm{~K}$ & $150 \mathrm{~K}$ & $300 \mathrm{~K}$ & $1000 \mathrm{~K}$ \\
\hline$\overline{\text { UX Ori }}$ & 27.9 & $1.9 \times 10^{-1}$ & $1.2 \times 10^{-2}$ & 1.0 & $6.8 \times 10^{-2}$ & $2.0 \times 10^{-2}$ \\
\hline HD 34282 & 13.8 & $9.7 \times 10^{-2}$ & $6.0 \times 10^{-3}$ & 10 & $\ldots$ & $\ldots$ \\
\hline HD 100453 & 1.9 & $1.4 \times 10^{-2}$ & $0.9 \times 10^{-3}$ & 0.1 & $0.9 \times 10^{-2}$ & $2.5 \times 10^{-3}$ \\
\hline HD 101412 & 5.3 & $3.7 \times 10^{-2}$ & $2.3 \times 10^{-3}$ & 0.2 & $1.6 \times 10^{-2}$ & $4.8 \times 10^{-3}$ \\
\hline HD 104237 & 5.0 & $3.6 \times 10^{-2}$ & $2.2 \times 10^{-3}$ & 0.1 & $0.8 \times 10^{-2}$ & $2.3 \times 10^{-3}$ \\
\hline HD 142666 & $\ldots$ & $\ldots$ & $\ldots$ & 0.2 & $1.0 \times 10^{-2}$ & $3.1 \times 10^{-3}$ \\
\hline HD 319139 & $\ldots$ & $\ldots$ & $\ldots$ & 0.2 & $1.2 \times 10^{-2}$ & $3.7 \times 10^{-3}$ \\
\hline
\end{tabular}

Upper mass limits assuming optically thin $\mathrm{H}_{2}$ in LTE.

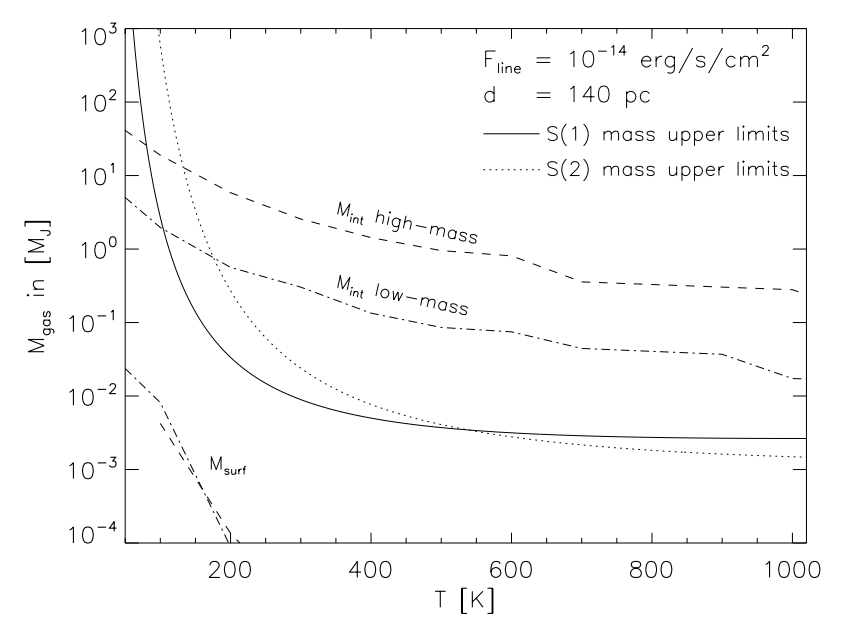

Fig. 4. Mass limits of optically thin $\mathrm{H}_{2}$ derived from $\mathrm{H}_{2} \mathrm{~S}(1)$ (solid line) and $\mathrm{H}_{2} \mathrm{~S}(2)$ (dotted line) as a function of the temperature for a line flux limit of $10^{-14} \mathrm{erg} \mathrm{s}^{-1} \mathrm{~cm}^{-2}$ for a source at a distance of $140 \mathrm{pc}$. Dashed and dot-dashed lines show the gas mass as function of the temperature for a Chiang \& Goldreich (1997) optically thick two-layer model for a low-mass $\left(M_{\text {DISK }}=0.02 M_{\odot}\right)$ and a high-mass $\left(M_{\text {DISK }}=0.11 M_{\odot}\right)$ disk assuming a gas-to-dust ratio of $100 . M_{\text {int }}$ is the mass of the interior layer. $M_{\text {surf }}$ is the mass of the surface layer. $\mathrm{H}_{2}$ emission arises only from the optically thin molecular gas in surface layer of the disk.

at a given temperature ${ }^{8}$. We see that the $\mathrm{S}(1)$ line sets constraints for the mass of optically thin $\mathrm{H}_{2}$ at temperatures $150 \mathrm{~K}<T<$ $500 \mathrm{~K}$. For higher temperatures the $\mathrm{S}(2)$ is a better tracer ${ }^{9}$.

\section{Discussion}

\subsection{Comparison with the gas mass of a two-layer disk model}

To understand the mass upper limits derived, we suppose that the disk has a two-layer structure (Chiang \& Goldreich 1997). In this disk model, the infrared excess in the SED is produced by the radiation of small dust grains in the superheated optically thin surface layer of the disk, in addition to the emission of cooler

${ }^{8}$ We calculated a set of CG97 disk models around a prototypical Herbig Ae star (see Sect. 5, and parameters of MWC 480 in Table 5) and found that the interior layer at $R>5 \mathrm{AU}$ at 17 and $12 \mu \mathrm{m}$ would be optically thin if the mass of a disk with a size of $100 \mathrm{AU}$ was smaller than $0.01 M_{J}$, if a gas-to-dust ratio of 100 and $T_{\text {gas }}=T_{\text {dust }}$ is assumed.

${ }^{9}$ For temperatures below $150 \mathrm{~K}$ the $\mathrm{S}(0)(J=2-0)$ line at $28 \mu \mathrm{m}$ provides better constraints to the optically thin gas than the $S(1)$ line. However, as the Earth's atmosphere is completely opaque at these wavelengths, the $S(0)$ line is only observable from space.
Table 5. Physical parameters of prototypical Herbig Ae/Be stars and their disks after Chiang et al. (2001).

\begin{tabular}{|c|c|c|}
\hline Parameter & $\begin{array}{l}\text { MWC } 480 \\
\text { high-mass }\end{array}$ & $\begin{array}{l}\text { HD } 36112 \\
\text { low-mass }\end{array}$ \\
\hline$T_{*}[\mathrm{~K}]$ & 8890 & 8465 \\
\hline$R_{*}\left[R_{\odot}\right]$ & 2.1 & 2.1 \\
\hline$M_{*}\left[M_{\odot}\right]$ & 2.3 & 2.2 \\
\hline$L_{*}\left[L_{\odot}\right]$ & 24.6 & 20.2 \\
\hline$d[\mathrm{pc}]$ & 140 & 150 \\
\hline$\Sigma_{0}\left[\mathrm{~g} \mathrm{~cm}^{-2}\right]$ & 8000 & 1000 \\
\hline$a_{0}[\mathrm{AU}]$ & 100 & 250 \\
\hline$H / h$ & 1.7 & 1.5 \\
\hline$p$ & 1.5 & 1.5 \\
\hline$M_{\text {DISK }}\left[M_{\odot}\right]$ & 0.11 & 0.02 \\
\hline
\end{tabular}

dust in the mid-plane. In the interior layer, the gas is thermalized by collisions with the dust grains, the column densities of $\mathrm{H}_{2}$ are larger than $10^{23} \mathrm{~cm}^{-2}$ and the optical depth is higher than one (see Fig. 6). The interior layer is optically thick in both gas and dust even at mid-infrared wavelengths. Emission lines from an optically thick medium are only observable when there are temperature gradients. The hot upper layer can provide emission lines against the cold mid-plane.

Note that the two layer model is an approximation of the real structure of the disk. In fact, in the uppermost zone of the surface layer, the gas is thermally decoupled from the dust, and due to intense X-ray or UV heating may well be ionized and at temperatures of the order of $10^{4} \mathrm{~K}$. This uppermost surface is above the region where the hydrogen is molecular. The $\mathrm{H}_{2}$ emission arises from a layer below this uppermost surface at about $A_{V}=$ 1 , or a hydrogen column about $10^{21}$ to $10^{23} \mathrm{~cm}^{-2}$ down from the surface, depending on the dust opacity. For the remainder of the discussion, one must keep in mind that when we speak about the disk surface layer, we refer specifically to the molecular gas layer of the disk's surface layer.

Using a two-layer disk model implementation (CGplus, Dullemond et al. 2001) with physical parameters aimed to fit the SED of the prototypical Herbig Ae/Be stars, we computed the expected amount of gas in the interior and surface layer as function of the temperature for disks of mass $0.11 M_{\odot}$ and $0.02 M_{\odot}$ (see Table 5 and Fig. 5) ${ }^{10}$. In Fig. 4 we present our results. The mass limits derived from the $\mathrm{H}_{2} \mathrm{~S}(1)$ and $\mathrm{S}(2)$ line observations

\footnotetext{
${ }^{10}$ In our models no puffed-up inner rim was used. We employed a model with a truncation radius at $T=5000 \mathrm{~K}$. We assumed that $T_{\text {gas }}=$ $T_{\text {dust }}$ and used a gas-to-dust ratio of 100 . For disk-models with $T_{\text {gas }} \neq$ $T_{\text {dust }}$ see for example, Kamp \& Dullemond (2004), Nomura \& Millar (2005) and Jonkheid et al. (2006).
} 

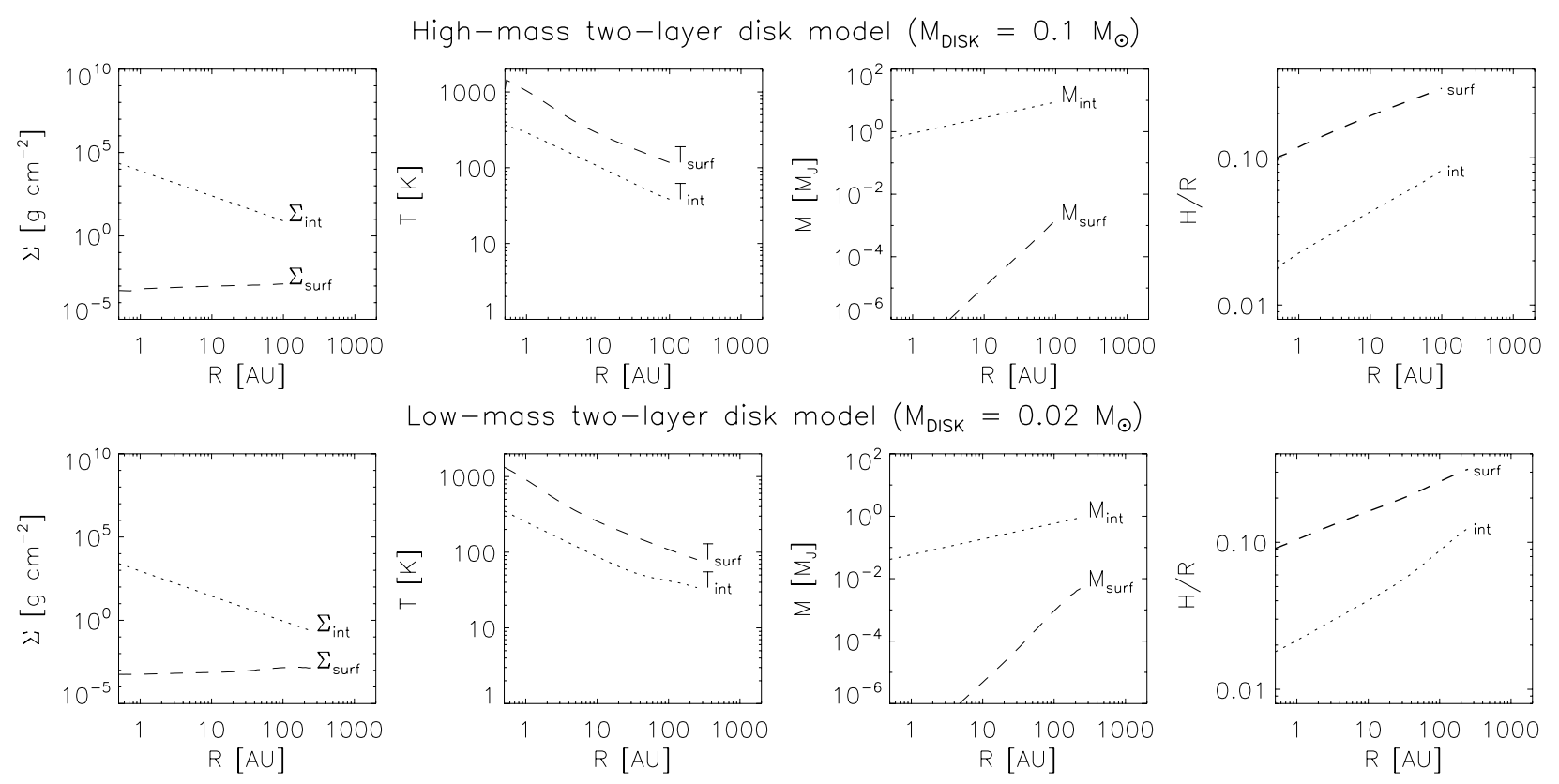

Fig. 5. Physical parameters of the two-layer disk model as a function of radius $(R)$. Upper panels: high-mass disk model $\left(M_{\mathrm{DISK}}=0.11 M_{\odot}, L_{*}=\right.$ $\left.24.6 L_{\odot}\right)$. Lower panels: low-mass disk model $\left(M_{\mathrm{DISK}}=0.02 M_{\odot}, L_{*}=20.2 L_{\odot}\right)$. The indices int and surf refer to the interior and the surface layer. $\Sigma$ is the surface mass density, $T$ the temperature, $M$ the disk mass and $H / R$ the scale height. See Table 5 for details of the model parameters.

are smaller than the amount of warm gas in the interior layer, but much larger than the amount of molecular gas in the surface layer. Figure 4 shows that the amount of gas in the surface layer is very small $\left(<10^{-2} M_{J} \sim 3 M_{\oplus}\right)$ and almost independent of the total mass of the disk. If the two-layer model is an adequate representation of the structure of the disk, the thermal flux levels of $\mathrm{H}_{2}$ mid-infrared emission are below the detection limit of the observations, because the mass of $\mathrm{H}_{2}$ in the surface layer is very small.

\subsection{Derivation of expected $S(1)$ and $S(2) H_{2}$ emission from the two-layer disk model}

Our observations are one of the most sensitive efforts to date to search for $\mathrm{H}_{2}$ emission from gas-rich protoplanetary disks (see Table 6). If the $\mathrm{H}_{2}$ emission is only produced from the upper layer of the disk, as the two layer model suggests, we would like to find out what flux levels we could expect from typical Herbig Ae/Be disks, assuming $T_{\text {gas }}=T_{\text {dust }}$ and a gas-to-dust ratio equal to 100 . For simplicity we first assume that the disk is viewed pole-on $\left(i=0^{\circ}\right)$. Here, we should note that the gas temperature can be higher than the dust temperature in the surface layer (e.g., Kamp \& Dullemond 2004; Nomura \& Millar 2005; Jonkheid et al. 2006; Glassgold 2007).

In the two-layer disk model, at each disk radius the $\mathrm{H}_{2}$ emission from the surface layer has to be observed on the top of the optically thick continuum of the interior layer, and the optically thin continuum of the dust at the surface layer. The total intensity (line plus continuum) emitted at each radius $(R)$ is given by

$I_{v}(R)=B_{v}\left(T_{\text {int }}\right) \mathrm{e}^{-\tau_{v_{\text {surf }}}}+B_{v}\left(T_{\text {surf }}\right)\left(1-\mathrm{e}^{-\tau_{v_{\text {surf }}}}\right)$.

Here, $B_{v}\left(T_{\text {int }}\right) \mathrm{e}^{-\tau_{v_{\text {surf }}}}$ is the contribution of the interior layer to the intensity. $B_{v}\left(T_{\text {int }}\right)$ is the black body emission of the interior layer at temperature $T_{\text {int }}$ seen through the surface layer optical depth $\left(\tau_{v_{\text {surf }}}=\tau_{v_{\text {dust surf }}}+\tau_{v_{\text {gas surf }}}\right)$. The contribution of the surface layer is $B_{v}\left(T_{\text {surf }}\right)\left(1-\mathrm{e}^{-\tau_{v_{\text {surf }}}}\right)$. It is the optically thin emission of the gas and dust in the surface layer at temperature $T_{\text {surf }}$.
The dust optical depth in the surface layer is given by

$$
\tau_{v_{\text {dust surf }}}=\kappa_{v_{\text {dust }}} \Sigma_{\text {dust }} \text { surf }
$$

where $\kappa_{v_{\text {dust }}}$ is the dust mass absorption coefficient ${ }^{11}$ at the frequency $v$, and $\Sigma_{\text {dust }}$ isurf the dust surface mass density in the surface layer of the disk given by the CG97 model.

The $\mathrm{H}_{2}$ gas optical depth in the surface layer at the frequencies $v$ in the vicinity of the $\mathrm{H}_{2}$ line at frequency $v_{0}$ is

$$
\tau_{v_{\text {gas surf }}}=\kappa_{v_{\text {line }}} \Sigma_{\text {gas }_{\text {surf }}} f_{2} \text {, }
$$

with $\Sigma_{\text {gas surf }_{\text {s }}}$ being the gas surface mass density in the disk surface layer (equal to 100 times $\Sigma_{\text {dust }}$ surf $)^{12}$, and $f_{2}$ the conversion factor from the total gas surface density $\Sigma_{\text {gas }_{\text {surf }}}$ to the $\mathrm{H}_{2}$-ortho or $\mathrm{H}_{2}$-para surface densities. Since $M_{\text {gas }}=M_{\text {ortho }}+M_{\text {para }}$, then $f_{2}=1 /(1+$ ortho/para $)$ for the $\mathrm{S}(2)$ line $\left(\right.$ a $\mathrm{H}_{2}$-para transition $)$ and $f_{2}=1 /\left(1+1 /(\right.$ ortho/para $)$ ) for the $\mathrm{S}(1)$ line (a $\mathrm{H}_{2}$-ortho transition). The gas mass absorption coefficient $\left(\kappa_{v_{\text {line }}}\right)$ in the vicinity of the $\mathrm{H}_{2}$ transition at frequency $v_{0}$ is given by

$\kappa_{v_{\text {line }}}=\frac{1}{2 m_{\mathrm{p}}} \frac{h v_{0}}{4 \pi}\left(x_{l} B_{\mathrm{lu}}-x_{u} B_{\mathrm{ul}}\right) \phi_{v}$.

Here, $2 m_{\mathrm{p}}$ is the mass of the $\mathrm{H}_{2}$ molecule (2 times the mass of the proton $\left.m_{\mathrm{p}}\right), x_{l}$ and $x_{u}$ are the lower and upper level population of the transition $J=u-l$, and $B_{\mathrm{lu}}$ and $B_{\mathrm{ul}}$ are the probabilities of absorption and emission.

We assumed that the gas is in LTE and derived the level populations using the Boltzmann equation. The line profile $\phi_{v}$ is assumed Gaussian with a $F W H M^{13}$ equal to $15 \mathrm{~km} \mathrm{~s}^{-1}$ :

$\phi_{v}=\frac{1}{\sigma_{v} \sqrt{\pi}} \mathrm{e}^{-\left(\frac{v-v_{0}}{\sigma_{v}}\right)^{2}}$,

\footnotetext{
11 We used the opacities by Laor \& Draine (1993).

12 Here we assume that all the gas is $\mathrm{H}_{2}$.

13 We checked that the optical depth is smaller than one with a $\phi$ with thermal width $\sigma_{\text {th }}=\sqrt{k T_{\mathrm{s}} /\left(2 m_{\mathrm{p}}\right)}$, therefore, $\phi$ could be assumed from the start with a width equal to the instrument resolution.
} 

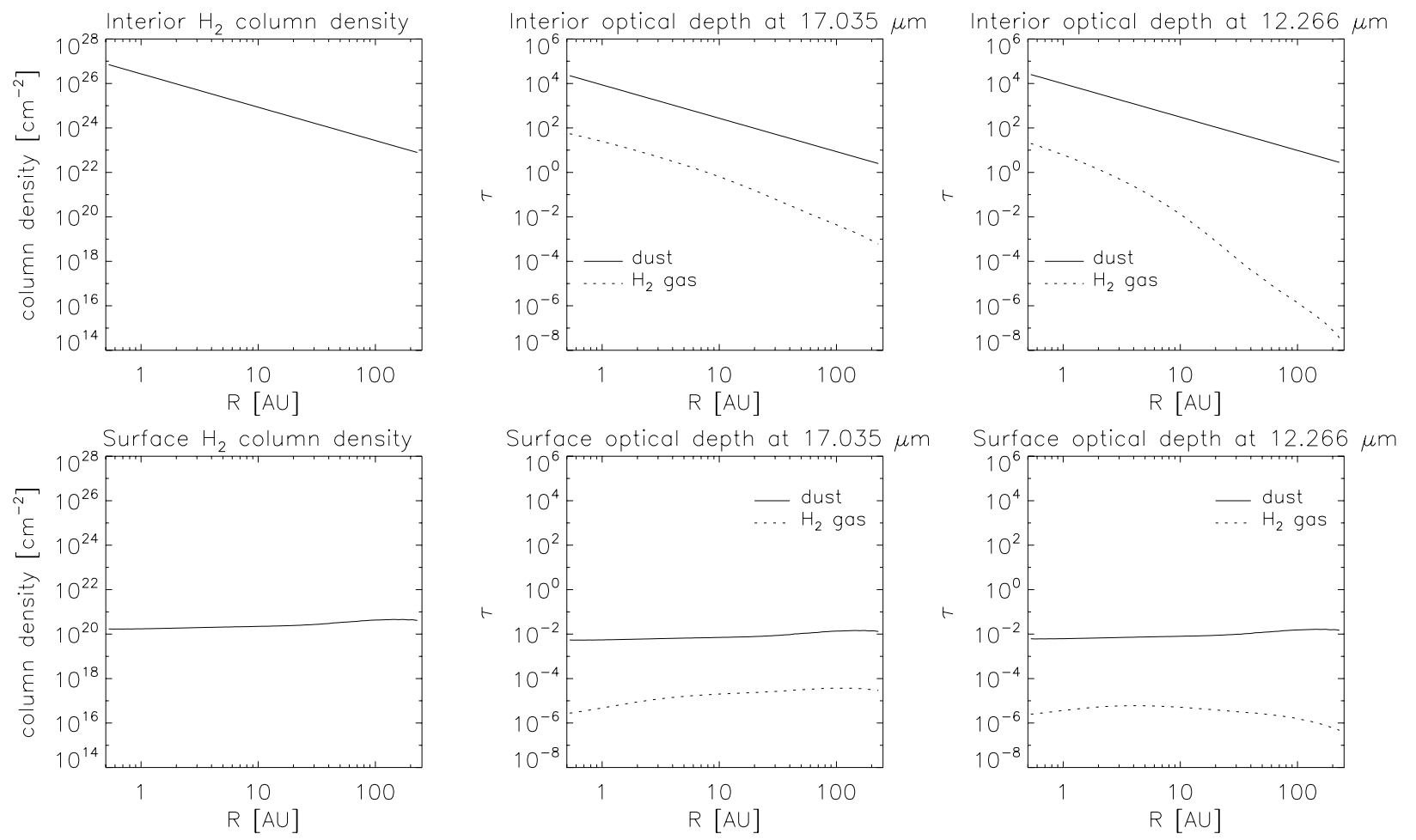

Fig. 6. Column density of $\mathrm{H}_{2}$ and optical depth of $\mathrm{H}_{2}$ and dust as a function of radius $R$ for the interior (upper panels) and the surface layer (lower panels $)$ in the two-layer low-mass disk model $\left(M_{\mathrm{DISK}}=0.02 M_{\odot}, L_{*}=20.2 L_{\odot}\right)$. A factor ten higher values in the $\mathrm{H}_{2}$ column density and optical depth of the interior layer are reached in the high-mass $\left(M_{\text {DISK }}=0.11 M_{\odot}, L_{*}=24.6 L_{\odot}\right)$ model. See Table 5 for details of the model parameters.

Table 6. Observational efforts to detect mid-IR $\mathrm{H}_{2}$ emission from gas-rich protoplanetary disks.

\begin{tabular}{lccccl}
\hline \hline Instrument & $\lambda / \Delta \lambda$ at $17 \mu \mathrm{m}$ & $\begin{array}{c}\mathrm{LW} \\
{\left[\mathrm{km} \mathrm{s}^{-1}\right]}\end{array}$ & $\begin{array}{c}\text { Beam } \\
{[\operatorname{arcsec} \times \operatorname{arcsec}]}\end{array}$ & $\begin{array}{c}\text { Flux \& flux limits } \\
{\left[\times 10^{-14} \mathrm{erg} \mathrm{s}^{-1} \mathrm{~cm}^{-2}\right]}\end{array}$ & Ref. \\
\hline VISIR & 21000 & 14 & $0.4 \times 0.4$ & $<0.5$ & C07 \\
& $10000^{a}$ & 30 & $0.4 \times 0.4$ & 2.4 & MZ07 \\
COMICS & 5000 & 60 & $0.6 \times 0.4$ & $<1.0^{b}$ & S05 \\
TEXES & 60000 & 5 & $2 \times 2$ & $<1.4^{c}$ & R02 \\
& 100000 & 3 & $0.4 \times 0.4$ & $1.1^{d}$ & B07 \\
MICHELLE & 15200 & 40 & $1 \times 1$ & $<6.7^{e}$ & SR03 \\
ISO-SWS & 2000 & 210 & $14 \times 27$ & $<0.8-40^{a, b, d}$ & T01 \\
SPITZER $^{f}$ & 600 & 500 & $5 \times 5$ & $0.32^{g}$ & L07 \\
\hline
\end{tabular}

${ }^{a}$ Observations of HD 97048 performed with a 0.75 " slit, therefore, the different resolution.

${ }^{b}$ The detection of $\mathrm{H}_{2}$ S(1) in HD 163296, MWC863, CQ Tau and LkCa15 by Thi et al. (2001) was not confirmed by Sako et al. (2005).

${ }^{c}$ The detection of $\mathrm{H}_{2} \mathrm{~S}(1)$ in CQ Tau and AB Aur by Thi et al. (2001) was not confirmed by Richter et al. (2002).

${ }^{d}$ Bitner et al. (2007) reported the detection of the $\mathrm{H}_{2} \mathrm{~S}(1), \mathrm{S}(2)$ and $\mathrm{S}(4)$ lines in AB Aur.

${ }^{e}$ The detection of $\mathrm{H}_{2}$ S(1) in HD 163296, AB Aur and GG Tau by Thi et al. (2001) was not confirmed by Sheret et al. (2003).

${ }^{f}$ From 76 sources studied one exhibits the $\mathrm{H}_{2} \mathrm{~S}(1)$ line and six the $\mathrm{H}_{2} \mathrm{~S}(2)$ line. None of the 8 HAeBes observed exhibit $\mathrm{H}_{2}$ mid-IR emission.

${ }^{g}$ Detection of the $\mathrm{H}_{2} \mathrm{~S}(1)$ line in the T Tauri star Sz102.

References: [B07] Bitner et al. (2007); [C07] Carmona et al. (this work); [L07] Lahuis et al. (2007); [MZ07] Martin-Zaïdi et al. (2007); [R02]

Richter et al. (2002); [S05] Sako et al. (2005); [SR03] Sheret et al. (2003); [T01] Thi et al. (2001).

where $\sigma_{v}=\left(v_{0} / c\right) * F W H M /(2 \sqrt{\ln 2})$. The expected flux $\mathrm{d} F_{v}(R)$ at each radius $(R)$ in the disk is given by

$\mathrm{d} F_{v}(R)=I_{v}(R) \mathrm{d} \Omega=I_{v}(R) \frac{2 \pi R \mathrm{~d} R}{d^{2}}$

with $\mathrm{d} \Omega$ being the solid angle, and $\mathrm{d} R$ the width of the region at a distance $R$ from the central object where the surface temperature is equal to $T_{\text {surf }}$ and the interior temperature is equal to $T_{\text {int }}$. The distance to the source is $d$.

Figure 7 shows two examples of the surface optical depth $\left(\tau_{v_{\text {surf }}}\right)$ and the continuum subtracted $\mathrm{S}(1)$ and $\mathrm{S}(2) \mathrm{H}_{2}$ predicted line fluxes for the regions with surface temperature $\sim 150 \mathrm{~K}(R \sim 47 \mathrm{AU}$ upper panel $)$ and $\sim 300 \mathrm{~K}(R \sim 7 \mathrm{AU}$ lower panel). We assumed for the calculation a face-on high-mass disk model at a distance of $140 \mathrm{pc}$. The column density of $\mathrm{H}_{2}$ in the interior layer of the disk is larger than $10^{23} \mathrm{~cm}^{-2}$. The total optical depth peaks at the $\mathrm{H}_{2} \mathrm{~S}(1)$ and $\mathrm{S}(2)$ rest wavelengths.

The contribution to the total flux in each line varies as a function of the distance to the central source. Since at each radius, the surface layer optical depth of dust and $\mathrm{H}_{2}$ gas is much smaller than one (see Fig. 6) Eq. (2) can be approximated by

$I_{v}(R)=B_{v}\left(T_{\text {int }}\right)+B_{v}\left(T_{\text {surf }}\right) \tau_{v_{\text {dust surf }}}+B_{v}\left(T_{\text {surf }}\right) \tau_{v_{\text {gas surf }}}$. 

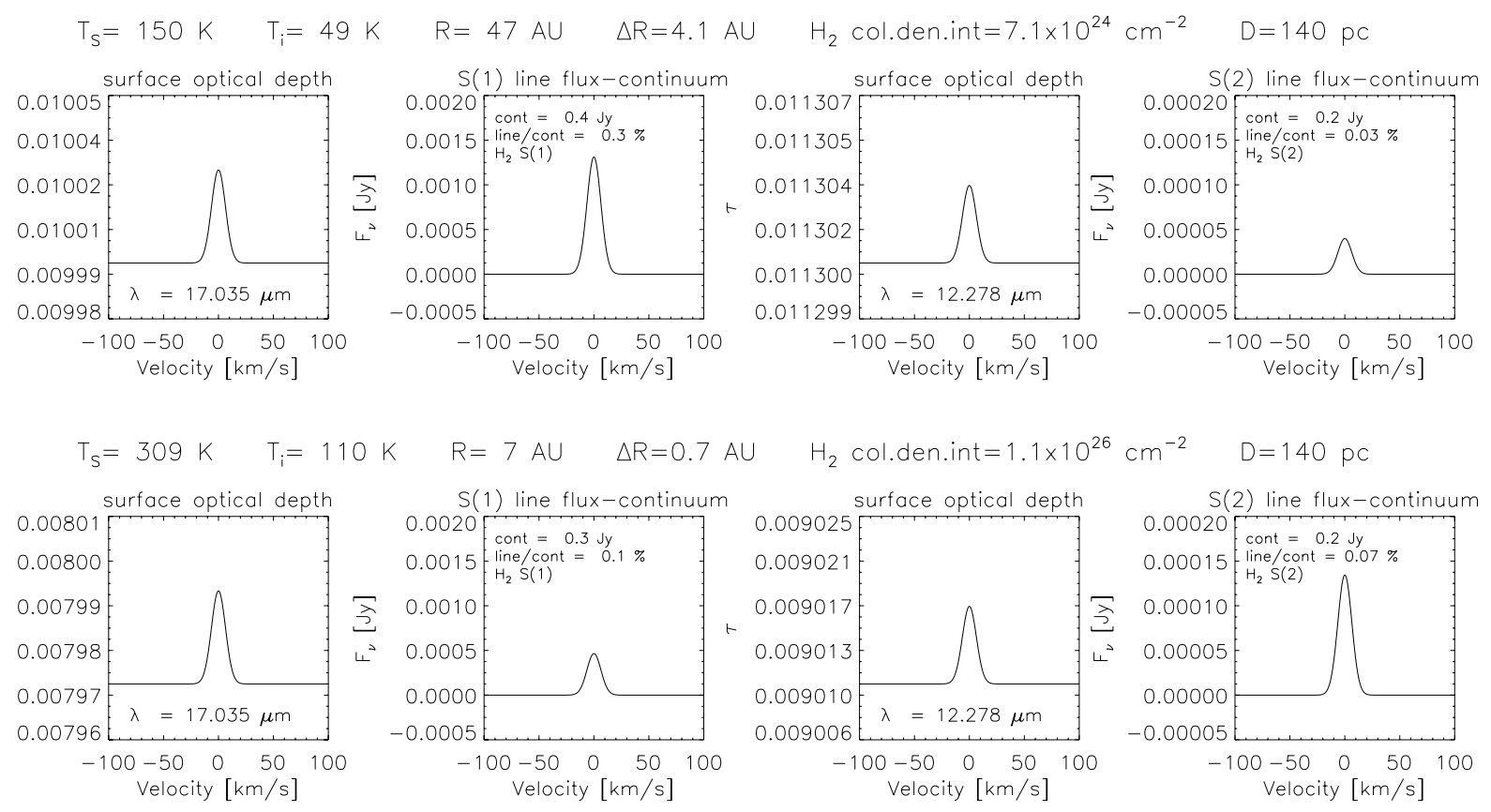

Fig. 7. Surface optical depth and predicted $\mathrm{H}_{2} \mathrm{~S}(1)$ and $\mathrm{S}(2)$ emission line flux for the regions where the surface temperature $\left(T_{\mathrm{s}}\right) \sim 150 \mathrm{~K}($ upper panel) and $\sim 300 \mathrm{~K}$ (lower panel) in the two-layer high-mass disk model $\left(M_{\mathrm{DISK}}=0.11 M_{\odot}, L_{*}=24.6 L_{\odot}\right)$. $\mathrm{T}_{i}$ is the interior temperature. An instrumental line $F W H M$ of $15 \mathrm{~km} \mathrm{~s}^{-1}$ was assumed. Note that the flux scale in the line flux-continuum plot is 10 times smaller in the $\mathrm{S}(2)$ line plot as in the $S(1)$ line plot.

In this way, the contributions to $I_{v}$ by the interior layer $B_{v}\left(T_{\text {int }}\right)$, by the dust in the surface $B_{v}\left(T_{\text {surf }}\right) \tau_{v_{\text {dust surf }}}$ and by the $\mathrm{H}_{2}$ line $B_{v}\left(T_{\text {surf }}\right) \tau_{v_{\text {gas surf }}}$ at the surface can be separated at each radius. Combining Eqs. (7) with (8), we can disentangle the different contributions to the flux $\mathrm{d} F_{v}$ as a function of the radius

$\mathrm{d} F_{v}(R)=\mathrm{d} F_{v_{\text {int }}}(R)+\mathrm{d} F_{v_{\text {dust surf }}}(R)+\mathrm{d} F_{v_{\text {gas surf }}}(R)$.

In the left panels of Fig. 8 we show the contributions, in percentage of the peak flux at each radius, by the interior layer $\left(\mathrm{d} F_{v_{\text {int }}}(R) / \mathrm{d} F_{v}(R)\right.$, solid line), the dust in the surface $\left(\mathrm{d} F_{v_{\text {dustsurf }}}(R) / \mathrm{d} F_{v}(R)\right.$, dashed lines) and the $\mathrm{H}_{2}$ emission $\left(\mathrm{d} F_{v_{\text {gas surf }}}(R) / \mathrm{d} F_{v}(R)\right.$, dotted lines). Up to a few AU, the main source of continuum emission is the dust in the interior layer; at larger radii, the principal source of continuum emission is the superheated dust in the surface layer. The $\mathrm{H}_{2}$ line contribution is always very small, typically less than $1 \%$ of the total continuum flux.

In the central panels of Fig. 8 we show the evolution of line peak flux minus the continuum as a function of radius. In the case of the $S(1)$ line the maximum contribution to the total flux is given by the region around 50-100 AU. In the case of the $\mathrm{S}(2)$ line the most important contribution to the line flux is made by material around 5-20 AU.

The total expected flux from the disk is the sum of the contributions of each radius. In the right panels of Fig. 8 we present the total expected line from the disk. The line strength over the continuum is less than $0.05 \mathrm{Jy}$ for the $\mathrm{S}(1)$ line and less than $0.005 \mathrm{Jy}$ for the $S(2)$ line. The total integrated flux of the line is of the order of $10^{-16} \mathrm{erg} \mathrm{s}^{-1} \mathrm{~cm}^{-2}$ for the $\mathrm{S}(1)$ line and $10^{-17} \mathrm{erg} \mathrm{s}^{-1} \mathrm{~cm}^{-2}$ for the $\mathrm{S}(2)$ line. These line flux levels are two orders of magnitude below the sensitivity limits of our observations.

We note that our $\mathrm{H}_{2}$ line flux predictions from the two-layer model only take into account thermal excitation of the gas, therefore, show the minimum amount of expected flux. If additional excitation mechanisms, such as X-rays or UV heating, are taken into account, the line fluxes are expected to be higher because the gas is hotter. In sophisticated models of the disk around the T Tauri star TW Hya $(d=56 \mathrm{pc})$ by Nomura et al. $(2005,2007)$, the predicted $\mathrm{H}_{2} \mathrm{~S}(1)$ and $\mathrm{S}(2)$ line fluxes are of the order of a few $10^{-15} \mathrm{erg} \mathrm{s}^{-1} \mathrm{~cm}^{-2}$ and the line-to continuum flux ratios are approximately $10^{-5}$. For a star at $140 \mathrm{pc}$ these line fluxes translate to a few $10^{-16} \mathrm{erg} \mathrm{s}^{-1} \mathrm{~cm}^{-2}$, fluxes similar to our twolayer model ${ }^{14}$. These theoretical $\mathrm{H}_{2}$ line fluxes are still two orders of magnitude below the detection limits of our observations. In addition, the achievement of line-to continuum flux ratios $<10^{-3}$ is a challenging task for present ground-based observations. In our observations, for example, the best line/continuum ratio achieved is $10^{-1}$.

\subsubsection{The effect of the disk inclination}

Until now, we assumed for simplicity that the disk is viewed pole-on. Now, if the disk is inclined by an angle $i$, the line profile $\phi_{v}$ used in Eq. (6) will be doppler-shifted for each parcel of gas that has a velocity component in the line of sight. For a parcel of gas located at radius $R$ and at azimuth ${ }^{15} \theta$, orbiting a star of mass $M_{\star}$, the doppler shift $\Delta v$ due to the Keplerian motion of the gas is

$\Delta v=\left(v_{0} / c\right) \sin (\theta) \sin (i) \sqrt{\frac{G M_{\star}}{R}}$,

with $c$ being the speed of light. The doppler-shifted line pro-

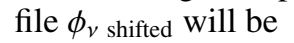

$\phi_{v \text { shifted }}=\frac{1}{\sigma_{v} \sqrt{\pi}} \mathrm{e}^{-\left(\frac{v-v_{0}+\Delta v}{\sigma_{v}}\right)^{2}}$.

${ }^{14}$ Note that T Tauri stars are less luminous than Herbig Ae/Be stars. The fluxes are similar because the Nomura models include additional sources of heating beyond dust-gas collisions.

15 We define $\theta=0$ as the angle where the velocity component of the motion is 0 in the line of sight. 


\section{High-mass disk model}
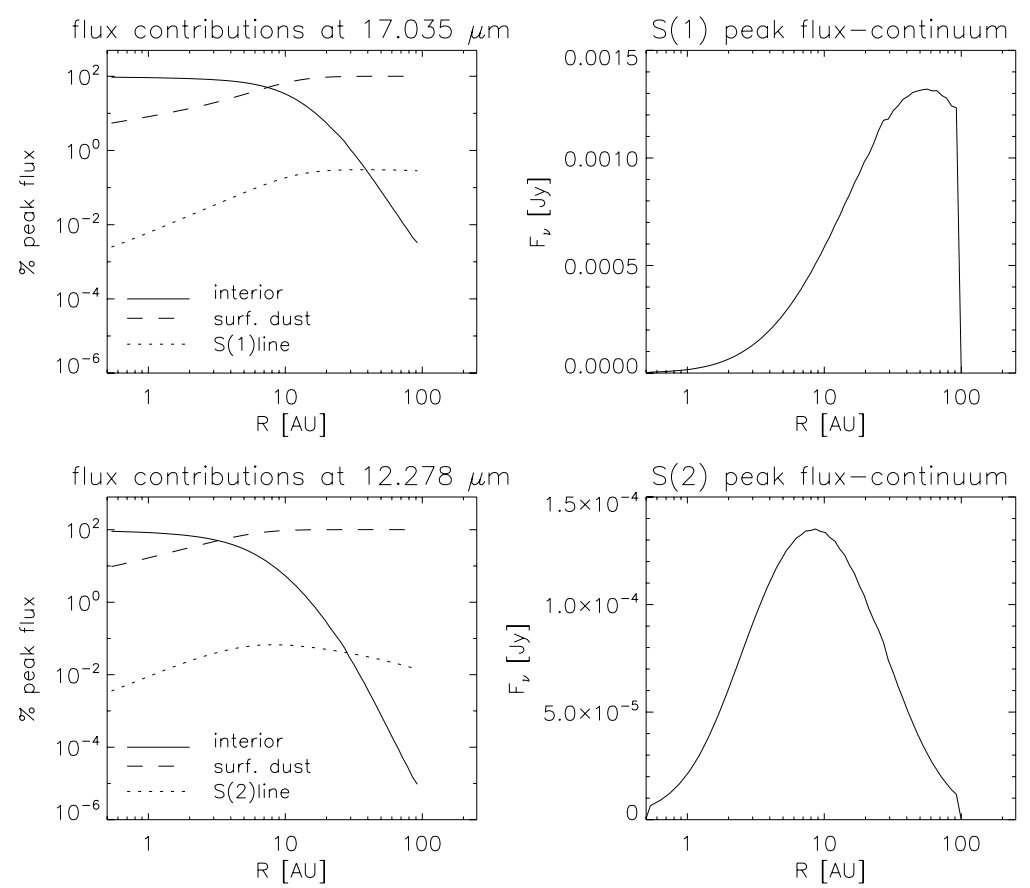

Low-mass disk model
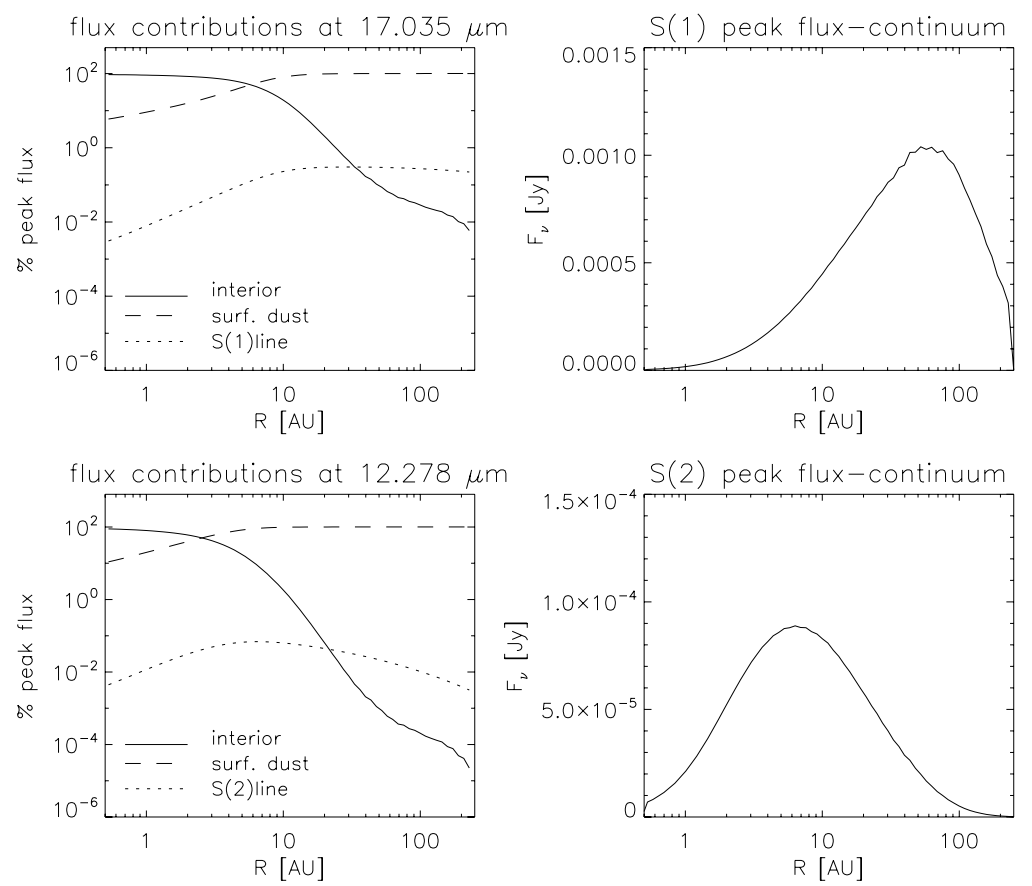
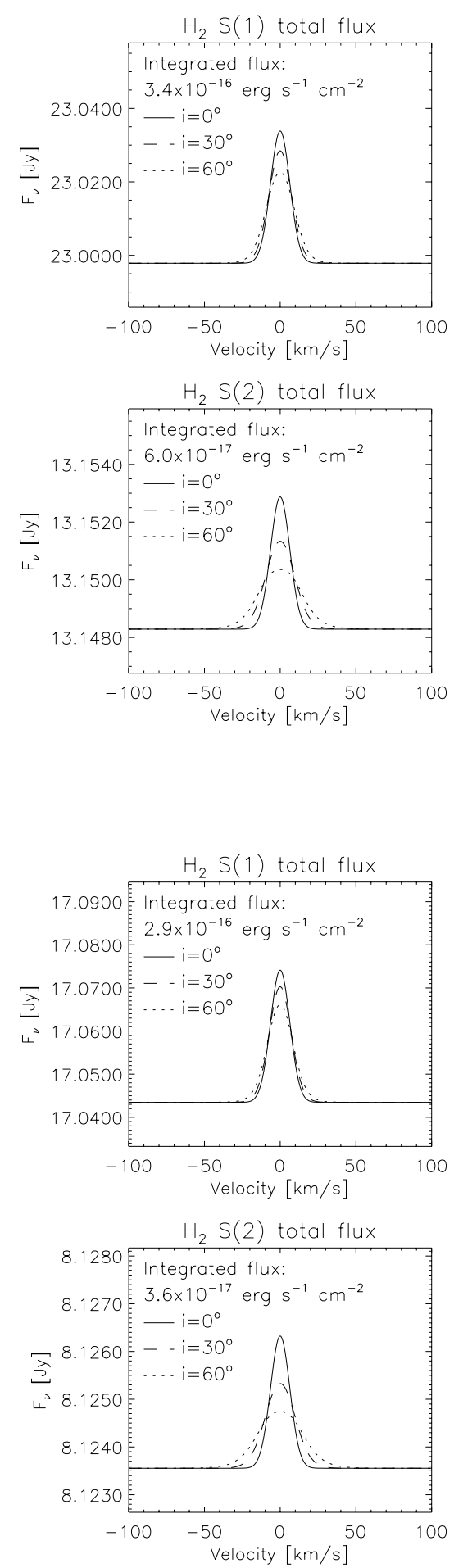

Fig. 8. Total S(1) and S(2) line fluxes for the two-layer high-mass disk model (upper six panels) and low-mass disk model (lower six panels). Left panels present the percentage contribution to the peak flux by the optically thick interior layer (solid line), the optically thin dust in the surface layer (dashed line) and the $\mathrm{H}_{2}$ line (dotted line) as a function of radius for a pole-on disk $\left(i=0^{\circ}\right)$. Central panels show the peak flux minus the continuum as a function of radius for pole-on disk. Right panels display the expected total $\mathrm{H}_{2}$ line flux for three disk inclinations: pole-on (solid line), $i=30^{\circ}$ (dashed line) and $i=60^{\circ}$ (dotted line).

Using Eq. (11) instead of Eq. (6) and employing Eqs. (3) to (8), the intensity $I(R, \theta, i)$ and the flux $\mathrm{d} F_{v}(R, \theta, i)$ emitted by each parcel of fluid of solid angle $(R \mathrm{~d} R \mathrm{~d} \theta) / \mathrm{d}^{2}$ is calculated. The expected total emission by the disk is the sum of the contributions of all the fluid parcels from $R_{\min }$ to $R_{\max }$, the inner and outer radius of the emitting region. Assuming that $R_{\min }=0.5 \mathrm{AU}$ and 


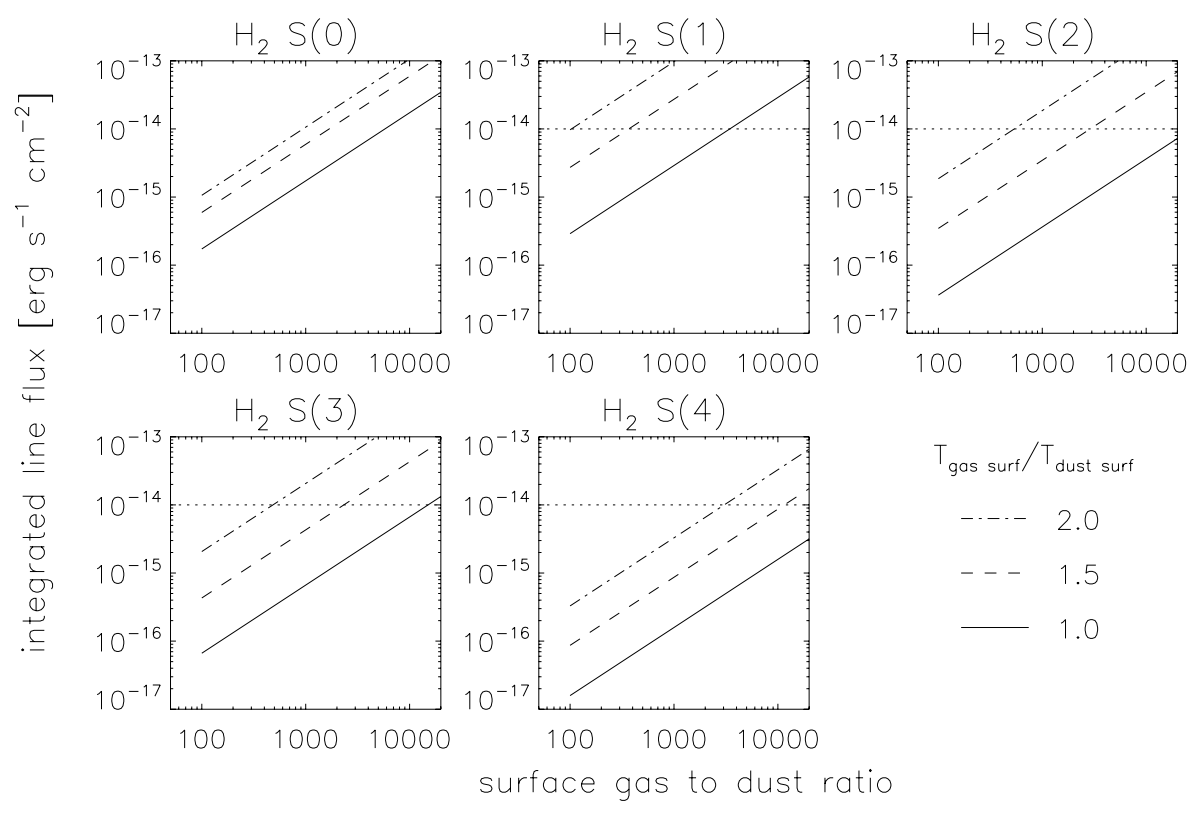

Fig. 9. $\mathrm{H}_{2} \mathrm{~S}(0), \mathrm{S}(1), \mathrm{S}(2), \mathrm{S}(3), \mathrm{S}(4)$ line fluxes for the two-layer low-mass disk model as a function of the gas-to-dust ratio in the surface layer for $T_{\text {gas }} / T_{\text {dust }}$ in the surface ranging from 1.0 to 2.0. The dotted horizontal line presents typical ground detection limits. The $\mathrm{S}(0)$ line is only observable from space. that $R_{\max }$ is the disk outer radius, we calculated the expected $\mathrm{H}_{2} \mathrm{~S}(1)$ and $\mathrm{H}_{2} \mathrm{~S}(2)$ line profiles for the high-mass and the lowmass disk at inclinations of 30 and 60 degrees. Our results are presented in the right panels of Fig. 8. The net effect of the inclination is the broadening of the line and a decrease of the peak flux. The $S(2)$ line is more affected than the $S(1)$ line by the inclination. This is due to the fact that $S(2)$ comes from warmer gas, which is closer in and therefore orbiting faster. Since most of the material responsible for the $\mathrm{S}(1)$ and $\mathrm{S}(2)$ emission is located at distances larger than a few AU (see central panels of Fig. 8) even at high inclinations, at our spectral resolution, the double peaked profile is not exhibited. This result suggests that higher temperature lines emitted in regions closer to the star (e.g., the $S(3)$ or $\mathrm{S}(4)$ lines) are more suitable for the investigation of inner disk dynamics.

\subsection{Detections and non-detections of $\mathrm{H}_{2}$ mid-IR emission from protoplanetary disks}

Our calculations of the expected thermal $\mathrm{H}_{2}$ line fluxes from CG97 disks around typical Herbig Ae/Be stars, and sophisticated models of $\mathrm{H}_{2}$ emission from disks around $\mathrm{T}$ Tauri stars by Nomura et al. $(2005,2007)$ show that the expected emission fluxes of $\mathrm{H}_{2}$ from optically thick disks in the mid-IR are very weak $\left(<10^{-16} \mathrm{erg} \mathrm{s}^{-1} \mathrm{~cm}^{-2}\right.$ for a source at $\left.140 \mathrm{pc}\right)$. These models provide a good explanation for the numerous non-detections of $\mathrm{H}_{2}$ mid-IR emission from optically thick disks reported so far (GG Tau, HD 163296, Richter et al. 2002; CQ Tau, Sheret et al. 2003; L551 IRS5, DG Tau, GW Ori, Richter et al. 2004; HD 163296, MWC 863, CQ Tau and LkCa 15, Sako et al. 2005; 62 T Tauri and 8 Herbig Ae/Be stars, Lahuis et al. 2007; UX Ori, HD 34282, HD 100453, HD 101412, HD 104237, HD 142666 and HD 319139, this work).

However, some detections of mid-IR $\mathrm{H}_{2}$ emission from disks have been reported in the Herbig Ae/Be stars AB Aur (Bitner et al. 2007) ${ }^{16}$ and HD 97048 (Martin-Zaïdi et al. 2007) ${ }^{17}$, and in the T Tauri stars Sz 102, EC 74, EC 82, Ced 110 IRS6, EC 92,

\footnotetext{
${ }^{16} \mathrm{H}_{2} \mathrm{~S}(1), \mathrm{S}(2)$ and $\mathrm{S}(4)$ lines.

${ }^{17} \mathrm{H}_{2} \mathrm{~S}(1)$ line.
}

and ISO-Cha 237 (Lahuis et al. 2007) ${ }^{18}$. An interesting question to address is the reason for the high $\mathrm{H}_{2}$ fluxes observed in these sources. Bitner et al. (2007), based on the gas temperature deduced from the observed lines $(670 \mathrm{~K})$ and constraints on the line emitting region (18 AU) by a line profile fit, concluded, by comparison to a disk model of AB Aur (Dullemond et al. 2001), that in order to explain the observed emission, an additional heating mechanism (X-rays or UV heating, e.g., Glassgold et al. 2007; Nomura et al. 2007) is needed to heat the gas in the disk upper layers (i.e. $T_{\text {gas surf }} / T_{\text {dust surf }}>1$ ). A second possibility to explain high $\mathrm{H}_{2}$ flux levels is to invoke a gas-to-dust ratio much larger than the canonical value of 100 in the surface layer of the disk (for example due to dust coagulation and sedimentation). Martin-Zaïdi et al. (2007) estimated the gas-to-dust ratio in HD 97048 from the detected $\mathrm{H}_{2} \mathrm{~S}(1)$ line and the dust mass required for producing the observed flux level in the continuum. They obtained gas-to-dust ratios ranging from 3000 to 14000 for gas temperatures between $150 \mathrm{~K}$ to $1000 \mathrm{~K}$.

In order to explore the influence of a change in the gas-todust ratio and the thermal decoupling of gas and dust in the surface layer, we calculated the expected $\mathrm{S}(0)$ to $\mathrm{S}(4) \mathrm{H}_{2}$ line fluxes from the low-mass disk model as a function of the surface gas-to-dust ratio for $T_{\text {gas surf }} / T_{\text {dust surf }}$ ranging from 1.0 to 2.0. We proceeded to use a simplified prescription. The output of a CG-plus model was taken as the disk surface mass density and temperature of the dust in the upper and interior layer. To obtain the molecular gas surface mass density in the upper layer, the dust surface mass density in this layer was multiplied by the gas-to-dust ratio. To obtain the molecular gas temperature in the upper layer, the dust temperature in this layer was multiplied by $T_{\text {gas surf }} / T_{\text {dust surf }}$. Our results are presented in Fig. 9.

As expected, an increase in the gas-to-dust ratio or

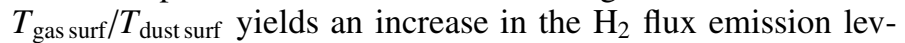
els. However, deviations from $T_{\text {gas surf }}=T_{\text {dust surf }}$ have a much larger impact on the increase of the strength of the $\mathrm{H}_{2}$ lines than the changes in the gas-to-dust ratio. For example, to obtain flux levels over $10^{-14} \mathrm{erg} \mathrm{s}^{-1} \mathrm{~cm}^{-2}$ in the $\mathrm{S}(1)$ line - sufficient to

\footnotetext{
${ }^{18} \mathrm{H}_{2} \mathrm{~S}(2)$ line, in addition the $\mathrm{H}_{2} \mathrm{~S}(1)$ and $\mathrm{S}(3)$ lines in $\mathrm{Sz} 102$ and the $\mathrm{H}_{2} \mathrm{~S}(3)$ line in EC 74.
} 
explain the detections by Bitner et al. and Mantin-Zaïdi et al. - it is just required that $T_{\text {gas surf }} / T_{\text {dust surf }}>2.0$. In contrast, to achieve similar S(1) flux levels the gas-to-dust ratio should increase by a factor of thirty. The most likely scenario is that in the molecular upper layer of the disk, both the gas temperature and the gas-todust ratio change. For example, a simultaneous increase in the gas-to-dust ratio $(>1000)$ and gas temperature $\left(T_{\text {gas surf }} / T_{\text {dust surf }}>\right.$ 2.0) can boost the flux levels of the $\mathrm{H}_{2} \mathrm{~S}(2)$ line to detectable levels. Therefore, the non detections in our observations suggest that in the observed disks, the molecular gas and dust in the surface layer has not significantly departed from thermal coupling and that the gas-to-dust ratio in the surface layer is not larger than 1000 .

One interesting aspect is that observations from space (Lahuis et al. 2007) report more numerous detections of the $\mathrm{S}(2)$ line than detections of the $\mathrm{S}(1)$ line, although in our LTE models the $S(1)$ line is in general stronger than the $S(2)$ line. Note that for a given temperature the amount of ortho $\mathrm{H}_{2}$ (responsible for the $\mathrm{S}(1)$ line) is higher than the amount of para $\mathrm{H}_{2}$ (responsible of the $\mathrm{S}(2)$ line). This discrepancy suggests that the level populations and the ortho/para ratio of the $\mathrm{H}_{2}$ producing the line in the observed sources are not in LTE. One additional piece of information suggesting that the $\mathrm{H}_{2}$ gas responsible for the line emission is heated by a mechanism other than collisions with dust, is the detection of $\mathrm{a}_{2} \mathrm{~S}(4)$ line $50 \%$ stronger than the $\mathrm{H}_{2} \mathrm{~S}(1)$ line in AB Aur (Bitner et al. 2007). In our LTE disk models the $\mathrm{H}_{2} \mathrm{~S}(4)$ line is always much weaker than the $\mathrm{H}_{2} \mathrm{~S}(1)$ line. Our results confirm the suggestion by Bitner et al. (2007) that in $\mathrm{AB}$ Aur the $\mathrm{H}_{2}$ emission is produced in a narrow region (at $18 \mathrm{AU})$, in which the gas has been excited to a high temperature $(T \simeq 670 \mathrm{~K})$. We speculate that this could be the location of shock-heated gas in the AB Aur disk.

\section{Summary and conclusions}

We observed a sample of nearby pre-main sequence stars with evidence for cold $(T<50 \mathrm{~K})$ gas disk reservoirs and searched for emission of the warm gas with $T>150 \mathrm{~K}$, which is expected to be present in the inner region of these disks. None of the targets show any evidence for $\mathrm{H}_{2}$ emission at $17.035 \mu \mathrm{m}$ or at $12.278 \mu \mathrm{m}$. From the $3 \sigma$ upper limits of the $\mathrm{H}_{2}$ line fluxes, we found stringent upper limits to the mass of optically thin warm $\mathrm{H}_{2}$. The disks contain less than a few tenths of Jupiter mass of optically thin $\mathrm{H}_{2}$ at $150 \mathrm{~K}$, and less than a few Earth masses of optically thin $\mathrm{H}_{2}$ at $300 \mathrm{~K}$ and higher temperatures.

Assuming that $T_{\text {gas }}=T_{\text {dust }}$ and a gas-to-dust ratio of 100, we compared our results to models of disks employing a Chiang \& Goldreich (1997) optically thick two-layer disk model of masses $0.02 M_{\odot}$ and $0.11 M_{\odot}$. The upper limits to the disk optically thin warm gas mass are smaller than the warm gas mass in the interior layer of the disk, but they are much larger than the amount of molecular gas expected to be in the surface layer. The amount of mass in the surface layer is very small $\left(<10^{-2} M_{J}\right)$ and almost independent of the total disk mass. We calculated the expected $\mathrm{H}_{2} \mathrm{~S}(1)$ and $\mathrm{H}_{2} \mathrm{~S}(2)$ line fluxes emitted from a two-layer disk for the low-mass and the high-mass cases assuming a distance of $140 \mathrm{pc}$, and LTE thermal emission. The predicted line fluxes of the two-layer disk model are of the order of $\sim 10^{-16}-10^{-17} \mathrm{erg} \mathrm{s}^{-1} \mathrm{~cm}^{-2}$, much smaller than the detection limits of our observations $\left(5 \times 10^{-15} \mathrm{erg} \mathrm{s}^{-1} \mathrm{~cm}^{-2}\right)$.

If the two-layer approximation to the structure of the disk is correct, we are essentially "blind" to most of the warm $\mathrm{H}_{2}$ in the disk because it is located in the optically thick interior layer of the disk. Our non-detections are explained because of the small flux levels expected from the little mass of $\mathrm{H}_{2}$ present in the optically thin surface layer. Naturally, the two-layer disk model is only an approximation of the real structure of a protoplanetary disk. The puffed-up inner rim, which could be an important contributor to the $\mathrm{H}_{2}$ emission, is not included in our models, and in reality there will be a smooth transition zone between the disk hot surface layer and the cool disk interior, which again could contribute significantly to the $\mathrm{H}_{2}$ emission. In addition, the surface layers of the disk are likely to have gas temperatures hotter than the dust temperatures or a gas-to-dust ratio higher than the canonical value of 100 . Both effects could potentially increase the $\mathrm{H}_{2}$ emission by significant amounts. We presented additional calculations in which the gas-to-dust ratio and the temperature of the molecular gas in the surface layer of the disk were increased. We showed that detectable $\mathrm{S}(1)$ and $\mathrm{S}(2) \mathrm{H}_{2}$ line flux levels can be achieved if $T_{\text {gas surf }} / T_{\text {dust surf }}>2$ and if the gas-to-dust ratio in the surface layer is greater than $1000 . \mathrm{H}_{2}$ emission levels are very sensitive to departures from the thermal coupling between the molecular gas and dust in the surface layer. Our results suggest that in the observed sources the molecular gas and the dust in the surface layer have not significantly departed from thermal coupling and that the gas-to-dust ratio in the surface layer is very likely lower than 1000. A definitive interpretation of our results awaits the development of future, more sophisticated models.

Acknowledgements. This research has made use of the SIMBAD database operated at CDS, Strasbourg, France. We would like to thank the staff of Paranal Observatory for performing our observations in service mode. We also would like to thank the referees, whose comments have helped to improve the content and presentation of our paper.

\section{References}

Acke, B., \& van den Ancker, M. E. 2004, A\&A, 426, 151

Acke, B., van den Ancker, M. E., \& Dullemond, C. P. 2005, A\&A, 436, 209

Beckwith, S. V. W., Sargent, A. I., Chini, R. S., \& Guesten, R. 1990, AJ, 99, 924 Bitner, M. A., Richter, M. J., Lacy, J. H., et al. 2007, ApJ, 661, L69 Boss, A. P. 1998, ApJ, 503, 923

Chen, C. H., Sargent, B. A., Bohac, C., et al. 2006, ApJS, 166, 351 Chiang, E. I., \& Goldreich, P. 1997, ApJ, 490, 368

Chiang, E. I., Joung, M. K., Creech-Eakman, M. J., et al. 2001, ApJ, 547, 1077 Cohen, M., Walker, R. G., \& Witteborn, F. C. 1999, LPI Contributions, 969, 5 de Zeeuw, P. T., Hoogerwerf, R., de Bruijne, J. H. J., Brown, A. G. A., \& Blaauw, A. 1999, AJ, 117, 354

Dent, W. R. F., Greaves, J. S., \& Coulson, I. M. 2005, MNRAS, 359, 663 Dullemond, C. P., Dominik, C., \& Natta, A. 2001, ApJ, 560, 957

Dullemond, C. P. 2002, A\&A, 395, 853

Dullemond, C. P., Hollenbach, D., Kamp, I., \& D’Alessio, P. 2007, in Protostars and Planets V., ed. B. Reipurth, D. Jewitt, \& K. Keil (Tucson: University of Arizona Press), 555

Dunkin, S. K., Barlow, M. J., \& Ryan, S. G. 1997, MNRAS, 286, 604

Dutrey, A., Guilloteau, S., \& Ho, P. 2007, in Protostars and Planets V., ed. B. Reipurth, D. Jewitt, \& K. Keil (Tucson: University of Arizona Press), 495 Glassgold, A. E., Najita, J. R., \& Igea, J. 2007, ApJ, 656, 515

Haisch, K. E., Jr., Lada, E. A., \& Lada, C. J. 2001, ApJ, 553, L153

Haisch, K. E., Jr., Jayawardhana, R., \& Alves, J. 2005, ApJ, 627, L57

Henning, T., Launhardt, R., Steinacker, J., \& Thamm, E. 1994, A\&A, 291, 546

Henning, T., Dullemond, C. P., Wolf, S., \& Dominik, C. 2006, in Planet Formation: Theory, Observations and Experiments, ed. H. Klahr, \& W. Brandner (Cambridge University Press), 112

Hollenbach, D., Gorti, U., Meyer, M., et al. 2005, ApJ, 631, 1180

Jensen, E. L. N., Mathieu, R. D., \& Fuller, G. A. 1996, ApJ, 458, 312

Jonkheid, B., Kamp, I., Augereau, J.-C., \& van Dishoeck, E. F. 2006, A\&A, 453, 163

Kamp, I., \& Dullemond, C. P. 2004, ApJ, 615, 991

Lagage, P. O., Pel, J. W., Authier, M., et al. 2004, The Messenger, 117, 12

Lahuis, F., van Dishoeck, E. F., Blake, G. A., et al. 2007, ApJ, 665, 492 
Laor, A., \& Draine, B. T. 1993, ApJ, 402, 441

Lundin, L. K. 2006, VLT VISIR Pipeline User Manual, VLT-MAN-ESO-195003852

Martin-Zaïdi, C., Lagage, P.-O., Pantin, E., \& Habart, E. 2007, ApJ, 666, L117

Meeus, G., Waters, L. B. F. M., Bouwman, J., et al. 2001, A\&A, 365, 476

Najita, J. R., Carr, J. S., Glassgold, A. E., \& Valenti, J. A. 2007, in Protostars and Planets V., ed. B. Reipurth, D. Jewitt, \& K. Keil (Tucso: University of Arizona Press), 507

Natta, A., Testi, L., Calvet, N., et al. 2007, in Protostars and Planets V., ed. B. Reipurth, D. Jewitt, \& K. Keil (Tucson: University of Arizona Press), 767

Nomura, H., \& Millar, T. J. 2005, A\&A, 438, 923

Nomura, H., Aikawa, Y., Tsujimoto, M., Nakagawa, Y., \& Millar, T. J. 2007 ApJ, 661, 334

Palla, F., \& Stahler, S. W. 1993, ApJ, 418, 414

Pascucci, I., Gorti, U., Hollenbach, D., et al. 2006, ApJ, 651, 1177

Piétu, V., Dutrey, A., \& Kahane, C. 2003, A\&A, 398, 565
Pollack, J. B., Hubickyj, O., Bodenheimer, P., et al. 1996, Icarus, 124, 62 Richter, M. J., Jaffe, D. T., Blake, G. A., \& Lacy, J. H. 2002, ApJ, 572, L161 Sako, S., Yamashita, T., Kataza, H., et al. 2005, ApJ, 620, 347

Sheret, I., Ramsay Howat, S. K., \& Dent, W. R. F. 2003, MNRAS, 343, L65 Siebenmorgen, R. L., Pantin, E., \& Sterzik, M. 2004, VLT VISIR User Manual.VLT-MAN-ESO-14300-3514

Sicilia-Aguilar, A., Hartmann, L. W., Fürész, G., et al. 2006, AJ, 132, 2135

Stempels, H. C., \& Gahm, G. F. 2004, A\&A, 421, 1159

Takahashi, J. 2001, ApJ, 561, 254

Thi, W. F., van Dishoeck, E. F., Blake, G. A., et al. 2001, ApJ, 561, 1074 van den Ancker, M. E., de Winter, D., \& Tjin A Djie, H. R. E. 1998, A\&A, 330, 145

Watson, A. M., Stapelfeldt, K. R., Wood, K., \& Ménard, F. 2007, in Protostars and Planets V., ed. B. Reipurth, D. Jewitt, \& K. Keil (Tucson: University of Arizona Press), 523

Wolniewicz, L., Simbotin, I., \& Dalgarno, A. 1998, ApJS, 115, 293 